\title{
Changes in food intake from 2005 to 2010 by a cohort of black rural and urban African men and women in the North West Province of South Africa: the PURE-NWP-SA study
}

\author{
Edelweiss Wentzel-Vilioen ${ }^{1, *}$, Ria Laubscher $^{2}$ and Hester $\mathrm{H} \mathrm{Vorster}^{1}$ \\ ${ }^{1}$ Centre of Excellence for Nutrition, Faculty of Health Sciences, North-West University, P/Bag X6003, Potchefstroom, \\ South Africa: ${ }^{2}$ South African Medical Research Council, Biostatistics Unit, Tygerberg, South Africa
}

Submitted 25 October 2017: Final revision received 12 June 2018: Accepted 2 July 2018: First published online 28 August 2018

\begin{abstract}
Objective: We have shown that nutrient intakes of rural and urban black Africans in the North West Province (NWP) of South Africa (SA) followed the typical nutrition transition pattern upon urbanization and modernization. The current study aimed to examine and report on the changes in food intakes from 2005 to 2010 in rural and urban black South Africans participating in the PURE-NWP-SA study.

Design/Setting/Subjects: The PURE-NWP-SA study recruited 2010 volunteers aged 35-70 years in 2005, from which detailed food intakes, measured with a validated quantified FFQ, for 1858 participants were available. In 2010, food intakes of a cohort of 1154 of these participants were measured.

Results: Median energy intake increased in men and women in both rural and urban areas from 2005 to 2010. Changes in food intake were interpreted keeping these changes in energy intake and the contribution of foods and food groups to total energy intake in mind. No 'new' foods were eaten in 2010, but more participants consumed certain foods and products in 2010 than in 2005. Beneficial changes were increased intakes of vegetables, fruit and milk in most groups. The contribution of cooked staple porridges and bread made from fortified maize and bread flour decreased and therefore also did their contribution to micronutrient intakes.

Conclusions: By promoting and supporting observed beneficial changes such as increased intakes of milk, vegetables and fruit by appropriate policies and educational interventions, it should be possible to steer the nutrition transition in this population into a positive direction.
\end{abstract}

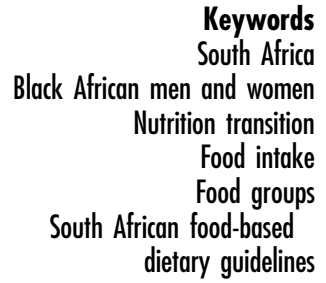

The global nutrition transition is often defined as the changes in nutrient consumption that occur when developing populations modernize and urbanize because of economic development and other social and lifestyle changes, leading to changes in the availability and affordability and thus exposure to new foods and products, including more processed and 'takeaway' foods ${ }^{(1,2)}$. The resultant changes in nutrient intakes are seen to be part of the epidemiological (health) transition, contributing to an increased risk of non-communicable diseases $(\mathrm{NCD})^{(1,2)}$. South Africa (SA), a low-middle-income country, is in the midst of a health transition characterized by a burden of communicable and non-communicable diseases as well as perinatal, maternal and injury-related disorders $^{(3)}$. There are sharp increases in overweight, obesity, hypertension, diabetes and CVD in South African populations without substantial improvements of undernutrition in children ${ }^{(3-5)}$. The South African Medical Research
Council $^{(6)}$ showed that both under- and overnutrition are risk factors for disability-adjusted life years and mortality in the South African population. This confirms previous research $^{(2)}$ that NCD emerged in sub-Saharan Africa at a faster rate and at a lower economic level than in industrialized countries in the past, before the battle against undernutrition has been won, leading to the phenomenon of a double burden of consequences of the coexistence of under- and overnutrition ${ }^{(2)}$.

We have previously illustrated that the diet followed by black South Africans in rural areas of the North West Province (NWP) does not meet micronutrient requirements ${ }^{(7-11)}$ and that both rural and urban black populations increased their added sugar intake, especially as sucrosesweetened beverages, from 2005 to $2010^{(12)}$. In an effort to address the micronutrient deficiencies in the South African population, the Department of Health (Directorate Nutrition) instituted a mandatory micronutrient fortification of 
the staples, maize meal and bread flour, in $2003^{(13)}$ that came into effect in 2004. Data on food intake per se of subpopulations in SA are scarce and a need has been identified to collect and publish food intake data of South Africans ${ }^{(14)}$.

To promote and strengthen the observed beneficial nutrition transition changes in micronutrient intakes of the PURE-NWP-SA participants with urbanization and modernization $^{(11)}$, and at the same time to prevent adverse changes in macronutrient intakes, it is necessary to know which foods were responsible for the reported changes in nutrient intakes ${ }^{(11)}$. Knowledge of these changes in food intake can then be applied in appropriate education programmes using the South African food-based dietary guidelines (SAFBDG) ${ }^{(15)}$ to promote optimal nutrition from diets that are adequate regarding micronutrients and at the same time protective against overnutrition and risk of NCD regarding the macronutrients. The purpose of the present paper is therefore to examine and report on the changes in food intakes from 2005 to 2010 in rural and urban black South Africans participating in the Prospective Urban and Rural Epidemiological (PURE-NWP-SA) study.

\section{Methods}

The PURE-NWP-SA study: participants and design

The participants were volunteers participating in the PURE-NWP-SA study. The PURE study is an international prospective cohort study tracking changing lifestyles, risk factors and chronic disease in urban and rural areas of seventeen countries in transition ${ }^{(16,17)}$. One of the South African legs of the PURE study is being conducted in a rural and an urban area of the NWP. The baseline study was conducted in 2005 and the first follow-up in 2010. The study design of the PURE-NWP-SA study has been described elsewhere ${ }^{(12,17,18)}$. Briefly, participants were recruited from four different sites (two in the rural area, two in the urban area) in the NWP of SA. The study was explained to the individuals and after voluntary and informed consent was obtained the questionnaires were completed. A total of 2010 participants were tested (about 500 in each community) in 2005, of which dietary data were available for 1858 participants. In the 5-year follow-up survey conducted in 2010, 1233 participants were included. Figure 1 provides a summary of the recruitment and follow-up procedures (adapted from Vorster et al. $\left.{ }^{(12)}\right)$.

\section{Measurements: questionnaires}

Standardized structured demographic, socio-economic, lifestyle and physical activity questionnaires of the international PURE study were used ${ }^{(16)}$. These questionnaires were adapted for the South African study where necessary. Sixteen volunteer fieldworkers were recruited from the communities where the study was executed and intensively trained to complete the questionnaires at the

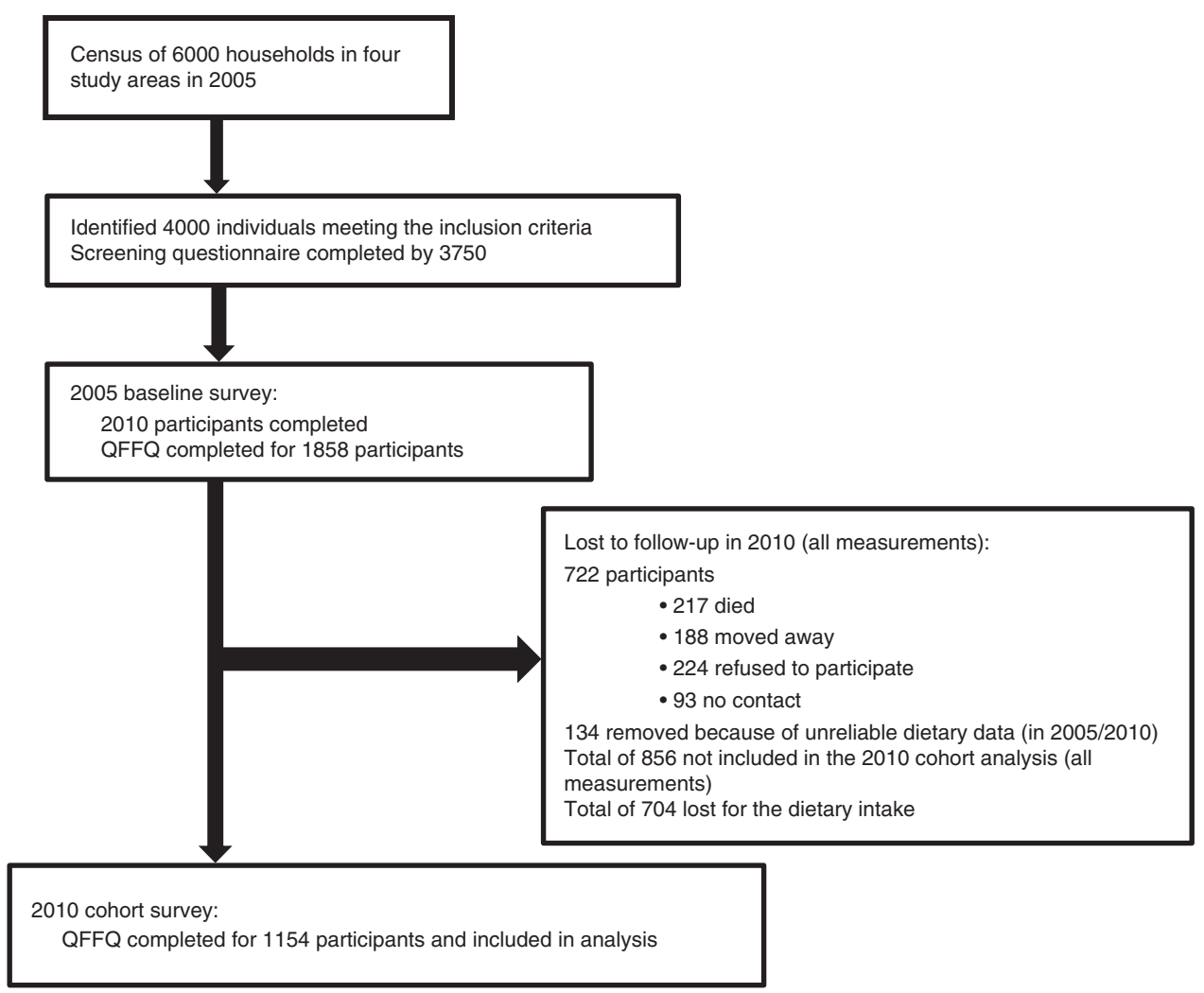

Fig. 1 Participant recruitment and follow-up from years 2005 to 2010 (adapted from Vorster et al. ${ }^{(12)}$ ). QFFQ, quantified FFQ 
study sites or participants' homes. The quantified FFQ (QFFQ) used to collect dietary intake data has been described elsewhere ${ }^{(11)}$. Briefly, a previously validated, culture-sensitive QFFQ was used to obtain dietary intakes covering the previous month ${ }^{(19-22)}$. Suitable tools, including a food-portion photograph book ${ }^{(23)}$ specifically developed and tested for this population, were used to quantify amounts and frequencies of foods eaten. The validation of the QFFQ included a comparison of intake results obtained with the questionnaire and with $7 d$ weighed records in a sample from the same communities participating in this study ${ }^{(20)}$. The food intake divided into seven food groups (milk; meat; fruit; vegetables; maize meal; bread; added sugar), as measured by the QFFQ and the $7 \mathrm{~d}$ weighed records, indicated that the QFFQ was valid also for these food groups ${ }^{(20)}$. The quantities of foods reported were converted to weights using standard tables ${ }^{(24)}$. When coding the QFFQ, mixed dishes, not available in the South African food composition tables ${ }^{(25)}$, were split into single ingredients according to recipes collected from the participants or 'standard', unpublished recipes obtained from the local communities, especially for traditional dishes such as Potchefstroom cookies, chakalaka (a salad of tinned beans, peppers and tomato) and ting (a fermented maize and/or sorghum porridge).
Food intake: approaches in presenting the data

Food intakes were categorized first into twelve food groups; Table 1 shows examples of the foods in each group, as well as the relevant SAFBDG ${ }^{(15,26-34)}$. Table 1 indicates that, generally, the first seven food groups may be regarded as the 'healthier' food groups with positive messages for consumption in the SAFBDG, while the last five groups may be regarded as the 'less healthy' choices with recommendations in the SAFBDG to limit intake or to use sparingly. In some food groups, both 'healthier' and 'less healthy choices' are possible (e.g. freshly cooked lean meat, chicken or fish in recommended quantities as the 'healthier choice' $v$. highly processed meats in larger-thanrecommended quantities as the 'unhealthy choice'). The twelve food groups in Table 1 are therefore based on the SAFBDG which aim to lead to optimum or adequate nutrition, while protecting against the development of risk factors of NCD.

Tables 2 and 3 show median intakes of energy and foods in each of the twelve food groups for men and women in rural and urban areas in both 2005 and 2010. Further groupings of individual food intakes or food group intakes (combinations of foods in grams) are shown in Tables 4 and 5 for those participants (men and women separately) who consumed these particular foods (by at least $5 \%$ of the participants in any group). For example,

Table 1 The content of the twelve food groups

\begin{tabular}{|c|c|c|c|}
\hline & Food group & Example of foods & Relevant SAFBDG \\
\hline 1 & Cooked porridge & $\begin{array}{l}\text { Maize meal, maltabella (sorghum porridge) and } \\
\text { oat porridge }\end{array}$ & $\begin{array}{l}\text { 'Make starchy foods part of most meals': a food-based } \\
\text { dietary guideline for South Africa }{ }^{(26)}\end{array}$ \\
\hline 2 & Starchy grains & $\begin{array}{l}\text { Breakfast cereals, bread, vetkoek (deep-fried } \\
\text { bread-type dough), samp (crushed maize), } \\
\text { maize rice, wheat rice, rice, macaroni and } \\
\text { pasta, starchy vegetables (potato, sweet } \\
\text { potato, mealies (corn-on-the-cob)) }\end{array}$ & $\begin{array}{l}\text { 'Make starchy foods part of most meals': a food-based } \\
\text { dietary guideline for South Africa }{ }^{(26)}\end{array}$ \\
\hline 3 & Vegetables & $\begin{array}{l}\text { All fresh and cooked vegetables like carrots, } \\
\text { tomato, onion, beetroot, butternut pumpkin }\end{array}$ & $\begin{array}{l}\text { 'Eat plenty of vegetables and fruit every day': a food-based } \\
\text { dietary guideline for South Africa }(27) \\
\text { Recommended daily intake for vegetables and fruit: } 400 \mathrm{~g} \\
(5 \times 80 \mathrm{~g})\end{array}$ \\
\hline 4 & Fruit & $\begin{array}{l}\text { Fresh fruit and fruit juices like apple, banana, } \\
\text { grapes }\end{array}$ & $\begin{array}{l}\text { 'Eat plenty of vegetables and fruit every day': a food-based } \\
\text { dietary guideline for South Africa } 27) \\
\text { Recommended daily intake for vegetables and fruit: } 400 \mathrm{~g} \\
(5 \times 80 \mathrm{~g})\end{array}$ \\
\hline 5 & Legumes & $\begin{array}{l}\text { Legume and legume products such as tinned } \\
\text { baked beans }\end{array}$ & $\begin{array}{l}\text { 'Eat dry beans, split peas, lentils and soya regularly': a } \\
\text { food-based dietary guideline for South Africa }{ }^{(28)}\end{array}$ \\
\hline 6 & Nuts and seeds & All nuts and seeds such as peanuts & No specific dietary guideline \\
\hline 7 & Milk & $\begin{array}{l}\text { All milk and milk products such as maas (thick } \\
\text { sour milk, often eaten with crumbly maize } \\
\text { porridge), yoghurt, cheese }\end{array}$ & $\begin{array}{l}\text { 'Have milk, maas or yoghurt every day': a food-based } \\
\text { dietary guideline for South Africa }\end{array}$ \\
\hline 8 & Animal protein foods & All meat, chicken, fish, and egg and products & $\begin{array}{l}\text { 'Fish, chicken, lean meat and eggs can be eaten daily': a } \\
\text { food-based dietary guideline for South Africa }{ }^{(30)}\end{array}$ \\
\hline 9 & Fats and oils & $\begin{array}{l}\text { All fats and oils and products (including ice } \\
\text { cream) }\end{array}$ & $\begin{array}{l}\text { The importance of the quality or type of fat in the diet: a } \\
\text { food-based dietary guideline for South Africa }{ }^{(31)}\end{array}$ \\
\hline 10 & $\begin{array}{l}\text { Added sugar } \\
\text { containing foods }\end{array}$ & $\begin{array}{l}\text { Sugar, syrups, sweets and refined products with } \\
\text { sugar added (sweet cookies, cakes, biscuits) }\end{array}$ & $\begin{array}{l}\text { Sugar and health: a food-based dietary guideline for South } \\
\text { Africa }^{(32)}\end{array}$ \\
\hline 11 & Savoury snacks & $\begin{array}{l}\text { Savoury snacks, dishes, sauces, seasoning and } \\
\text { products }\end{array}$ & $\begin{array}{l}\text { 'Use salt and foods high in salt sparingly': a food-based } \\
\text { dietary guideline for South Africa }{ }^{(33)}\end{array}$ \\
\hline 12 & Alcohol & $\begin{array}{l}\text { Alcoholic drinks like homemade beer, } \\
\text { commercial beer }\end{array}$ & $\begin{array}{l}\text { 'If you drink alcohol, drink sensibly'. Is this guideline still } \\
\text { appropriate? }{ }^{(34)}\end{array}$ \\
\hline
\end{tabular}

SAFBDG, South African food-based dietary guideline. 
Table 2 Daily energy intake, energy distribution of macronutrients and intakes of twelve selected food groups of male participants according to rural/urban residence in 2005 and 2010; PURE-NWP-SA study

\begin{tabular}{|c|c|c|c|c|c|c|c|c|c|c|}
\hline & \multicolumn{5}{|c|}{ Rural men ( $n$ 186) } & \multicolumn{5}{|c|}{ Urban men (n 202) } \\
\hline & \multicolumn{2}{|r|}{2005} & \multicolumn{2}{|r|}{2010} & \multirow[b]{2}{*}{$P$ value ${ }^{*}$} & \multicolumn{2}{|r|}{2005} & \multicolumn{2}{|r|}{2010} & \multirow[b]{2}{*}{$P$ value } \\
\hline & Median & P25-P75 & Median & P25-P75 & & Median & P25-P75 & Median & P25-P75 & \\
\hline Energy intake (MJ) & $6 \cdot 9$ & $5 \cdot 6-8 \cdot 8$ & 9.7 & $6 \cdot 95-13 \cdot 8$ & $<0.001$ & 9.9 & $7 \cdot 2-12 \cdot 6$ & $13 \cdot 7$ & $10 \cdot 5-17.9$ & $<0.001$ \\
\hline$\%$ of TE from protein & $10 \cdot 6$ & $9 \cdot 8-11 \cdot 6$ & 11.3 & $9 \cdot 5-13.5$ & 0.005 & 12.5 & $11.6-13.5$ & $12 \cdot 7$ & $11 \cdot 3-14.3$ & 0.483 \\
\hline$\%$ of TE from total fat & $17 \cdot 8$ & $13 \cdot 6-23 \cdot 1$ & 21.5 & $15 \cdot 7-28 \cdot 9$ & $<0.001$ & 24.9 & $21 \cdot 6-29 \cdot 4$ & $26 \cdot 3$ & $22 \cdot 1-31 \cdot 3$ & 0.329 \\
\hline $\begin{array}{l}\% \text { of TE from total } \\
\text { carbohydrate }\end{array}$ & 63.8 & $58 \cdot 1-69 \cdot 7$ & 59.5 & $51 \cdot 6-66 \cdot 5$ & $<0.001$ & $56 \cdot 1$ & $52 \cdot 0-60 \cdot 6$ & $54 \cdot 3$ & $48 \cdot 4-59 \cdot 7$ & 0.011 \\
\hline $\begin{array}{l}\text { Cooked porridge (e.g. } \\
\text { maize meal, oats) } \\
\text { and maize-based } \\
\text { drinks }(\mathrm{g})\end{array}$ & $700 \cdot 0$ & $500 \cdot 0-1000 \cdot 0$ & $621 \cdot 4$ & $350 \cdot 0-928.6$ & 0.0649 & $539 \cdot 3$ & $357 \cdot 1-857 \cdot 1$ & $678 \cdot 6$ & $392 \cdot 9-1150 \cdot 0$ & 0.0014 \\
\hline $\begin{array}{l}\text { Starchy grains } \\
\text { (cereals, bread, } \\
\text { vetkoek), starchy } \\
\text { vegetables } \\
\text { (potato, sweet } \\
\text { potato, corn) }(\mathrm{g})\end{array}$ & $192 \cdot 1$ & $115 \cdot 0-276 \cdot 4$ & $231 \cdot 7$ & $120 \cdot 3-348 \cdot 2$ & 0.002 & $240 \cdot 6$ & $160 \cdot 6-326 \cdot 4$ & $335 \cdot 3$ & $230 \cdot 8-483 \cdot 1$ & $<0.001$ \\
\hline Fresh vegetables $(\mathrm{g})$ & $40 \cdot 1$ & $24 \cdot 1-58 \cdot 6$ & 39.4 & $14 \cdot 6-86.5$ & 0.0271 & $70 \cdot 9$ & $48 \cdot 4-103 \cdot 6$ & $87 \cdot 8$ & $49 \cdot 5-141 \cdot 7$ & $<0.001$ \\
\hline $\begin{array}{l}\text { Fresh fruit and fruit } \\
\text { juices }(\mathrm{g})\end{array}$ & $15 \cdot 4$ & $0.0-28.6$ & $56 \cdot 1$ & $16 \cdot 9-142 \cdot 9$ & $<0.001$ & $65 \cdot 0$ & $25 \cdot 0-128 \cdot 6$ & $152 \cdot 4$ & $66 \cdot 9-265 \cdot 7$ & $<0.001$ \\
\hline $\begin{array}{l}\text { Legumes and legume } \\
\text { products }(\mathrm{g})\end{array}$ & 0.0 & $0.0-5 \cdot 7$ & $9 \cdot 1$ & $0.0-33.3$ & $<0.001$ & $2 \cdot 1$ & $0.0-20.9$ & $18 \cdot 6$ & $1 \cdot 1-48 \cdot 6$ & $<0.001$ \\
\hline Nuts and seeds (g) & & 0 & & 0 & & 1.4 & $0.0-5 \cdot 7$ & 0.0 & $0.0-5 \cdot 7$ & 0.6659 \\
\hline $\begin{array}{l}\text { Milk and milk } \\
\text { products }(\mathrm{g})\end{array}$ & $7 \cdot 5$ & $0.0-71.4$ & $109 \cdot 6$ & $21 \cdot 4-222 \cdot 9$ & $<0.001$ & 102.9 & $54 \cdot 6-184 \cdot 3$ & 181.5 & $88 \cdot 6-301 \cdot 1$ & $<0.001$ \\
\hline $\begin{array}{l}\text { All meat, chicken, fish, } \\
\text { and egg and } \\
\text { products }(\mathrm{g})\end{array}$ & $47 \cdot 8$ & $28 \cdot 3-73 \cdot 6$ & $97 \cdot 2$ & $49 \cdot 4-162 \cdot 5$ & $<0.001$ & $131 \cdot 1$ & $90 \cdot 4-191 \cdot 0$ & $190 \cdot 5$ & $127 \cdot 1-302 \cdot 4$ & $<0.001$ \\
\hline $\begin{array}{l}\text { All fats and oils and } \\
\text { products (including } \\
\text { ice cream) (g) }\end{array}$ & $9 \cdot 4$ & $4 \cdot 7-14 \cdot 7$ & $16 \cdot 1$ & $4 \cdot 4-30 \cdot 1$ & $<0.001$ & $19 \cdot 5$ & $12 \cdot 9-30 \cdot 1$ & $23 \cdot 7$ & $9 \cdot 0-46.5$ & 0.0186 \\
\hline $\begin{array}{l}\text { Sugar, syrups, sweets } \\
\text { and refined products } \\
\text { with sugar added } \\
\text { (sweet cookies, } \\
\text { cakes, biscuits) (g) }\end{array}$ & $25 \cdot 0$ & $15 \cdot 3-49 \cdot 9$ & $102 \cdot 1$ & $37 \cdot 1-210 \cdot 0$ & $<0.001$ & $85 \cdot 0$ & $45 \cdot 6-148 \cdot 7$ & 141.4 & $68 \cdot 3-272 \cdot 6$ & $<0.001$ \\
\hline $\begin{array}{l}\text { Savoury snacks, } \\
\text { dishes, sauces, } \\
\text { seasoning and } \\
\text { products }(\mathrm{g})\end{array}$ & & 0 & $3 \cdot 3$ & $0.0-15 \cdot 0$ & $<0.001$ & $11 \cdot 7$ & $0.9-31.4$ & 24.5 & $6.9-57.4$ & $<0.001$ \\
\hline Alcoholic drinks (g) & $143 \cdot 6$ & $0.0-1428.6$ & 14.4 & $0.0-707 \cdot 1$ & $<0.001$ & 362.5 & $0.0-1071 \cdot 4$ & 211.6 & $0.0-857.1$ & 0.1135 \\
\hline
\end{tabular}

sunflower oil intake is reported as a single food item, while all yellow vegetables and all green leafy vegetables are grouped together. Faber et al. identified options and methods available to group foods together for analysis purposes ${ }^{(35)}$. We therefore followed a combination of a food group and an FBDG approach while keeping foods with a low contribution to micronutrient intake (e.g. added sugar) separate. The SAFBDG describe milk intake as a separate guideline and hence our decision to keep it separate from animal protein intake ${ }^{(29)}$. The sequence of the food items is based on the percentage of consumers among the rural men/women in 2005. In addition, foods (or food groups) that are known to be associated with either a positive or negative health outcome were kept separate to facilitate statistical analysis in future. Tables 6-9 show the contribution of specific foods or food groups to total energy intake.

\section{Statistical analyses}

As previously reported, participants with energy intakes of $\geq 30000$ or $\leq 3000 \mathrm{~kJ} / \mathrm{d}$ were excluded ${ }^{(11,12)}$. The statistical software package Stata version 14 was used for the analysis. Since the food intake data were skewed, results are reported as median and 25th-75th percentile. Wilcoxon's signed-rank test was used to test for changes in food intakes based on the twelve food groups between 2005 and 2010 for each of the four groups (by gender and study site). To test for significant changes for the individual food items (as it was not always the same individuals who 
Table 3 Daily energy intake, energy distribution of macronutrients and intakes of twelve selected food groups of female participants according to rural/urban residence in 2005 and 2010; PURE-NWP-SA study

\begin{tabular}{|c|c|c|c|c|c|c|c|c|c|c|}
\hline & \multicolumn{5}{|c|}{ Rural women ( $n$ 411) } & \multicolumn{5}{|c|}{ Urban women ( $n$ 355) } \\
\hline & \multicolumn{2}{|r|}{2005} & \multicolumn{2}{|r|}{2010} & \multirow[b]{2}{*}{$P$ value* } & \multicolumn{2}{|r|}{2005} & \multicolumn{2}{|r|}{2010} & \multirow[b]{2}{*}{$P$ value* } \\
\hline & Median & P25-P75 & Median & P25-P75 & & Median & P25-P75 & Median & P25-P75 & \\
\hline Energy intake (MJ) & $6 \cdot 2$ & $5 \cdot 0-7 \cdot 6$ & $9 \cdot 1$ & $6 \cdot 9-12 \cdot 8$ & $<0.001$ & $9 \cdot 0$ & $6.5-11.6$ & $11 \cdot 7$ & $8.9-14.9$ & $<0.001$ \\
\hline$\%$ of TE from protein & $10 \cdot 9$ & $9 \cdot 9-12 \cdot 0$ & 11.1 & $9 \cdot 6-12 \cdot 9$ & 0.326 & 12.5 & $11 \cdot 3-13.5$ & 12.5 & $11 \cdot 1-14 \cdot 3$ & 0.025 \\
\hline$\%$ of TE from total fat & $20 \cdot 3$ & $15 \cdot 6-24 \cdot 5$ & $22 \cdot 9$ & $17 \cdot 7-30 \cdot 0$ & $<0.001$ & $28 \cdot 4$ & $23.7-31.9$ & $27 \cdot 7$ & $22 \cdot 8-32 \cdot 3$ & 0.335 \\
\hline $\begin{array}{l}\% \text { of TE from total } \\
\text { carbohydrate }\end{array}$ & $66 \cdot 7$ & $61 \cdot 3-71 \cdot 9$ & $61 \cdot 7$ & $53 \cdot 8-67 \cdot 7$ & $<0.001$ & $55 \cdot 7$ & $51 \cdot 1-60 \cdot 2$ & $54 \cdot 1$ & $49 \cdot 5-59 \cdot 8$ & 0.039 \\
\hline $\begin{array}{l}\text { Cooked porridge (e.g. } \\
\text { maize meal, oats) and } \\
\text { maize-based drinks (g) }\end{array}$ & $700 \cdot 0$ & $500 \cdot 0-928 \cdot 6$ & $650 \cdot 0$ & $400 \cdot 0-942 \cdot 9$ & 0.3063 & $448 \cdot 1$ & $324 \cdot 9-714 \cdot 3$ & $507 \cdot 1$ & $303 \cdot 6-781.4$ & 0.0756 \\
\hline $\begin{array}{l}\text { Starchy grains (cereals, } \\
\text { bread, vetkoek), starchy } \\
\text { vegetables (potato, } \\
\text { sweet potato, corn) (g) }\end{array}$ & $176 \cdot 7$ & $115 \cdot 7-260 \cdot 0$ & $235 \cdot 6$ & $145 \cdot 2-358 \cdot 6$ & $<0.001$ & $230 \cdot 0$ & $152 \cdot 1-316 \cdot 7$ & $333 \cdot 3$ & $218 \cdot 0-451 \cdot 4$ & $<0.001$ \\
\hline Fresh vegetables $(\mathrm{g})$ & $46 \cdot 4$ & $31 \cdot 8-61 \cdot 7$ & $58 \cdot 1$ & $25 \cdot 8-103 \cdot 6$ & $<0.001$ & $75 \cdot 6$ & $54 \cdot 6-112 \cdot 0$ & 105.5 & $66 \cdot 1-170 \cdot 3$ & $<0.001$ \\
\hline $\begin{array}{l}\text { Fresh fruit and fruit } \\
\text { juices }(\mathrm{g})\end{array}$ & $22 \cdot 4$ & $8 \cdot 4-43 \cdot 6$ & $58 \cdot 6$ & $19 \cdot 3-153 \cdot 6$ & $<0.001$ & $75 \cdot 7$ & $30 \cdot 0-142 \cdot 9$ & $164 \cdot 3$ & $94 \cdot 3-297 \cdot 6$ & $<0.001$ \\
\hline $\begin{array}{l}\text { Legumes and legume } \\
\text { products }(\mathrm{g})\end{array}$ & 0.0 & $0.0-11.4$ & $12 \cdot 9$ & $0 \cdot 0-40 \cdot 0$ & $<0.001$ & $10 \cdot 0$ & $0.0-28.9$ & 21.4 & $7 \cdot 1-46 \cdot 4$ & $<0.001$ \\
\hline Nuts and seeds (g) & & 0 & 0.0 & $0.0-1 \cdot 1$ & 0.0636 & 0.0 & $0.0-5 \cdot 0$ & 0.0 & $0.0-4.6$ & 0.0333 \\
\hline Milk and milk products $(\mathrm{g})$ & 8.0 & $0.0-60.0$ & 111.4 & $16 \cdot 6-221 \cdot 4$ & $<0.001$ & $124 \cdot 0$ & $63 \cdot 4-219 \cdot 3$ & $170 \cdot 0$ & $77 \cdot 9-254 \cdot 0$ & $<0.001$ \\
\hline $\begin{array}{l}\text { All meat, chicken, fish, and } \\
\text { egg and products }(\mathrm{g})\end{array}$ & $45 \cdot 1$ & $26 \cdot 3-72 \cdot 6$ & $77 \cdot 6$ & $37 \cdot 1-138 \cdot 6$ & $<0.001$ & $119 \cdot 6$ & $83 \cdot 4-177 \cdot 3$ & 173.9 & $102 \cdot 3-247 \cdot 2$ & $<0.001$ \\
\hline $\begin{array}{l}\text { All fats and oils and } \\
\text { products (including ice } \\
\text { cream) (g) }\end{array}$ & $9 \cdot 1$ & $4.9-15 \cdot 9$ & $18 \cdot 1$ & $7 \cdot 1-37 \cdot 1$ & $<0.001$ & $23 \cdot 7$ & $13 \cdot 9-41 \cdot 4$ & $23 \cdot 1$ & $10 \cdot 4-45 \cdot 7$ & 0.4678 \\
\hline $\begin{array}{l}\text { Sugar, syrups, sweets } \\
\text { and refined products } \\
\text { with sugar added } \\
\text { (sweet cookies, cakes, } \\
\text { biscuits) (g) }\end{array}$ & $32 \cdot 7$ & $14 \cdot 3-72 \cdot 4$ & $101 \cdot 4$ & $40 \cdot 9-196 \cdot 0$ & $<0.001$ & 99.4 & $64 \cdot 1-163 \cdot 4$ & $164 \cdot 4$ & $66 \cdot 0-305 \cdot 1$ & $<0.001$ \\
\hline $\begin{array}{l}\text { Savoury snacks, dishes, } \\
\text { sauces, seasoning and } \\
\text { products }(\mathrm{g})\end{array}$ & 0.0 & $0.0-3.3$ & $7 \cdot 2$ & $0.6-22.9$ & $<0.001$ & 17.4 & $4 \cdot 0-41 \cdot 1$ & $26 \cdot 2$ & $9.3-53.5$ & $<0.001$ \\
\hline Alcoholic drinks (g) & & 0 & & 0 & 0.0035 & 0.0 & $0.0-428.6$ & 0.0 & $0.0-214.3$ & 0.0377 \\
\hline
\end{tabular}

consumed a food item in 2005 and 2010) is a challenge. We used quartile regression (median specifically) to test whether there was a change in the gram intake of a food from 2005 to 2010, between rural and urban, for men and women separately. These are shown in Fig. 2 for the individual food groups consumed by at least $90 \%$ of the participants and contributing at least $10 \%$ to total energy intake (for both genders and in 2005 and 2010), namely: (i) cooked porridge (e.g. maize meal, maltabella, oats); (ii) samp, maize rice, wheat rice, rice, macaroni and spaghetti; (iii) sugar, white and brown; and (iv) bread, rolls, vetkoek.

We previously reported the baseline mean characteristics in 2005 of the cohort and of those participants lost to follow-up ${ }^{(11)}$, as shown in Table 10. As mentioned, food intakes (grams per day) are reported in Tables 2 to 5. Table 11 presents the percentage of consumers meeting the recommended intakes for fruit and vegetables and milk and milk products in 2005 and 2010 based on the SAFBDG $^{(27,29)}$.

\section{Results}

\section{Comparison of the cobort with those lost from the study}

Dietary intake data of 1858 participants were measured in 2005. Table 10 shows the characteristics (in 2005) of the 1154 cohort participants who could be followed up in 2010 compared with the baseline characteristics of the 856 participants (of the total original recruited group of 2010 participants in 2005) lost to follow-up. The latter were slightly (but significantly) younger, with lower BMI, HDL-cholesterol and total energy intake. The percentage of men in the group lost to follow-up was also significantly higher.

\section{Comparison of food intakes in 2005 and 2010}

The top rows of Tables 2 to 5 give the energy distribution of total energy intake between protein, fat and carbohydrate. These data illustrate the slight but significant 


\begin{tabular}{|c|c|c|c|c|c|c|c|c|c|c|c|c|}
\hline & \multicolumn{6}{|c|}{ Rural men $(n 186)$} & \multicolumn{6}{|c|}{ Urban men ( $n$ 202) } \\
\hline & \multicolumn{3}{|c|}{2005} & \multicolumn{3}{|c|}{2010} & \multicolumn{3}{|c|}{2005} & \multicolumn{3}{|c|}{2010} \\
\hline & $\%^{*}$ & Median & P25-P75 & $\%^{*}$ & Median & P25-P75 & $\%^{*}$ & Median & P25-P75 & $\%^{*}$ & Median & P25-P75 \\
\hline Energy intake (MJ) & - & 6.9 & $5 \cdot 6-8 \cdot 8$ & - & 9.7 & $6.95-13 \cdot 8$ & - & 9.9 & $7 \cdot 2-12 \cdot 6$ & - & 13.7 & $10.5-17.9$ \\
\hline$\%$ of TE from protein & - & $10 \cdot 6$ & $9.8-11 \cdot 6$ & - & 11.3 & $9.5-13.5$ & - & 12.5 & $11.6-13.5$ & - & $12 \cdot 7$ & $11 \cdot 3-14 \cdot 3$ \\
\hline$\%$ of TE from total fat & - & 17.8 & $13 \cdot 6-23 \cdot 1$ & - & 21.5 & $15 \cdot 7-28 \cdot 9$ & - & 24.9 & $21 \cdot 6-29 \cdot 4$ & - & $26 \cdot 3$ & $22 \cdot 1-31 \cdot 3$ \\
\hline$\%$ of TE from total carbohydrate & - & 63.8 & $58 \cdot 1-69.7$ & - & 59.5 & $51.6-66.5$ & - & $56 \cdot 1$ & $52 \cdot 0-60.6$ & - & 54.3 & $48 \cdot 4-59.7$ \\
\hline Cooked porridge (e.g. maize meal, oats) (g) & 99.5 & $700 \cdot 0$ & $500 \cdot 0-1000 \cdot 0$ & $100 \cdot 0$ & $550 \cdot 0$ & $287 \cdot 6-900 \cdot 0$ & $100 \cdot 0$ & 532.1 & $357 \cdot 1-857 \cdot 1$ & 99.5 & 571.4 & $317 \cdot 8-1007.5$ \\
\hline Sunflower oil (g) & 96.8 & $5 \cdot 3$ & $2 \cdot 9-8.1$ & 94.1 & 2.6 & $1 \cdot 0-6 \cdot 1$ & 100.0 & 9.4 & $6 \cdot 1-12 \cdot 4$ & 97.5 & 3.6 & $1.9-6 \cdot 1$ \\
\hline $\begin{array}{l}\text { Samp, maize rice, wheat rice, rice, macaroni, } \\
\text { spaghetti }(\mathrm{g})\end{array}$ & $96 \cdot 2$ & $85 \cdot 7$ & $46 \cdot 4-142 \cdot 9$ & $96 \cdot 8$ & 69.0 & $33 \cdot 6-120 \cdot 3$ & 95.5 & $71 \cdot 4$ & $35 \cdot 7-108 \cdot 9$ & $97 \cdot 0$ & $75 \cdot 0$ & $41 \cdot 6-129.9$ \\
\hline Sugar, white and brown (g) & 95.7 & 21.9 & $12 \cdot 6-30 \cdot 6$ & $97 \cdot 3$ & 33.9 & $16 \cdot 3-66 \cdot 0$ & 98.5 & $25 \cdot 1$ & $13 \cdot 7-45 \cdot 7$ & 97.5 & 34.4 & $17 \cdot 3-60 \cdot 0$ \\
\hline Bread, rolls, vetkoek (g) & 94.6 & $80 \cdot 0$ & $41.8-131.4$ & 98.4 & $74 \cdot 3$ & $40 \cdot 0-135 \cdot 7$ & 97.0 & $114 \cdot 3$ & $68 \cdot 6-171 \cdot 1$ & $97 \cdot 0$ & $122 \cdot 9$ & $63 \cdot 6-195 \cdot 0$ \\
\hline $\begin{array}{l}\text { Chicken, turkey, goose, duck, cooked, including } \\
\text { Kentucky (g) }\end{array}$ & 94.6 & $12 \cdot 9$ & $7 \cdot 1-25 \cdot 8$ & $95 \cdot 2$ & $19 \cdot 3$ & $10 \cdot 1-35 \cdot 7$ & 98.0 & $38 \cdot 2$ & $21 \cdot 6-58 \cdot 0$ & 96.5 & $62 \cdot 1$ & $33 \cdot 0-91 \cdot 3$ \\
\hline $\begin{array}{l}\text { Yellow and red vegetables (e.g. red peppers, } \\
\text { carrots, pumpkin, beetroot) }(\mathrm{g})\end{array}$ & 91.9 & $14 \cdot 3$ & $8 \cdot 6-22 \cdot 1$ & 88.2 & $15 \cdot 8$ & $5 \cdot 9-38 \cdot 2$ & 91.6 & $25 \cdot 0$ & $13 \cdot 6-38 \cdot 6$ & 93.6 & $26 \cdot 4$ & $15 \cdot 8-45 \cdot 2$ \\
\hline Coffee and tea $(\mathrm{g})$ & 88.7 & $300 \cdot 0$ & $300 \cdot 0-300 \cdot 0$ & $95 \cdot 2$ & $600 \cdot 0$ & $300 \cdot 0-900 \cdot 0$ & 97.5 & $220 \cdot 0$ & $157 \cdot 1-321 \cdot 4$ & 96.0 & 352.9 & $214 \cdot 3-600 \cdot 0$ \\
\hline $\begin{array}{l}\text { Other cooked vegetables (e.g. parsnips, green } \\
\text { beans, peas) (g) }\end{array}$ & $88 \cdot 2$ & $4 \cdot 0$ & $2 \cdot 1-7 \cdot 4$ & $86 \cdot 0$ & 6.9 & $2 \cdot 1-14 \cdot 1$ & 99.5 & $10 \cdot 7$ & $6 \cdot 9-16 \cdot 1$ & 98.0 & $19 \cdot 4$ & $9 \cdot 2-35 \cdot 9$ \\
\hline $\begin{array}{l}\text { Green vegetables (e.g. spinach, cabbage, } \\
\text { coleslaw, broccoli) (g) }\end{array}$ & $87 \cdot 6$ & $17 \cdot 1$ & $8 \cdot 6-25 \cdot 7$ & 86.6 & 10.5 & $4 \cdot 2-25 \cdot 7$ & 98.0 & $16 \cdot 0$ & $9 \cdot 3-27 \cdot 3$ & $89 \cdot 1$ & $17 \cdot 8$ & $8 \cdot 6-33 \cdot 0$ \\
\hline Tomato, cooked and raw $(\mathrm{g})$ & 79.0 & 4.9 & $3.4-8.0$ & $75 \cdot 8$ & 4.3 & $1.7-13.5$ & 98.0 & $12 \cdot 4$ & $7 \cdot 4-21 \cdot 1$ & 94.1 & 11.8 & $5 \cdot 8-22 \cdot 1$ \\
\hline Canned fish (e.g. pilchards, sardines, tuna) (g) & 71.0 & $6 \cdot 0$ & $4 \cdot 1-12 \cdot 0$ & 54.8 & 4.3 & $1.6-11.4$ & 68.8 & 8.9 & $4.4-17.7$ & 58.9 & 6.0 & $3 \cdot 0-17.7$ \\
\hline White-flesh fruit (e.g. apples, bananas, pears) (g) & $66 \cdot 1$ & $22 \cdot 9$ & $13 \cdot 4-33 \cdot 6$ & 83.9 & 48.6 & $19 \cdot 3-124 \cdot 5$ & 83.2 & $45 \cdot 7$ & $23 \cdot 9-86 \cdot 4$ & 93.6 & $114 \cdot 3$ & $55 \cdot 7-190 \cdot 0$ \\
\hline $\begin{array}{l}\text { Starchy vegetables (e.g. potato, sweet potato, } \\
\text { mealies) }(\mathrm{g})\end{array}$ & 61.3 & 13.7 & $8 \cdot 6-24 \cdot 9$ & $81 \cdot 2$ & $26 \cdot 4$ & $9 \cdot 5-65 \cdot 4$ & $96 \cdot 0$ & 28.6 & $12 \cdot 1-50 \cdot 0$ & $95 \cdot 0$ & 37.9 & $20 \cdot 7-76 \cdot 0$ \\
\hline Beef, cooked + meatballs (g) & $57 \cdot 0$ & 11.6 & $5 \cdot 0-14 \cdot 3$ & 71.5 & $12 \cdot 9$ & $6 \cdot 4-25 \cdot 7$ & $82 \cdot 2$ & $26 \cdot 3$ & $14 \cdot 3-43 \cdot 6$ & 78.7 & 28.6 & $11 \cdot 4-48 \cdot 4$ \\
\hline Beer, commercial and homemade (g) & 55.9 & 1171.4 & $285 \cdot 7-2821 \cdot 4$ & 51.1 & $678 \cdot 6$ & $171 \cdot 4-1285 \cdot 7$ & 69.3 & $670 \cdot 0$ & $321.4-1392.9$ & 61.9 & 535.7 & $285 \cdot 7-1321 \cdot 4$ \\
\hline Non-dairy creamer, milk blend $(\mathrm{g})$ & $51 \cdot 1$ & $6 \cdot 0$ & $4.0-8 \cdot 0$ & 44.1 & 12.0 & $6.0-18.9$ & 14.9 & $4 \cdot 3$ & $2.9-8.6$ & $19 \cdot 3$ & 8.6 & $4.3-18.0$ \\
\hline Milk, full-fat (g) & $50 \cdot 0$ & $52 \cdot 1$ & $35 \cdot 7-140$ & 79.0 & 120.0 & $51 \cdot 4-253 \cdot 6$ & 89.6 & $97 \cdot 7$ & $60 \cdot 0-157 \cdot 1$ & 88.6 & $184 \cdot 3$ & $87 \cdot 9-260 \cdot 0$ \\
\hline Eggs $(g)$ & 47.8 & 14.9 & $11 \cdot 1-29 \cdot 7$ & 62.4 & 28.6 & $14 \cdot 3-60 \cdot 7$ & 77.7 & $22 \cdot 3$ & $14.9-44.0$ & 86.6 & 29.8 & $20 \cdot 9-59 \cdot 4$ \\
\hline $\begin{array}{l}\text { Organ meats (e.g. liver, kidney, tripe, offal, heart, } \\
\text { giblets, lung, tongue, etc.) (g) }\end{array}$ & $44 \cdot 1$ & 11.4 & $5 \cdot 7-17 \cdot 1$ & $60 \cdot 2$ & $12 \cdot 9$ & $4 \cdot 9-25 \cdot 7$ & $78 \cdot 2$ & $17 \cdot 1$ & $8 \cdot 6-28 \cdot 6$ & $80 \cdot 7$ & 21.5 & $8 \cdot 8-44 \cdot 3$ \\
\hline $\begin{array}{l}\text { Meat products (e.g. boerewors, biltong, ham, } \\
\text { corned meat, viennas, frankfurter, salami, } \\
\text { patties) (g) }\end{array}$ & $26 \cdot 3$ & 7.7 & $3.9-10 \cdot 6$ & $61 \cdot 8$ & $8 \cdot 6$ & $4 \cdot 3-25 \cdot 7$ & $93 \cdot 1$ & $15 \cdot 4$ & $9 \cdot 3-29 \cdot 7$ & $89 \cdot 6$ & 21.4 & $10 \cdot 0-41 \cdot 4$ \\
\hline Carbonated cold drinks (g) & $26 \cdot 3$ & 37.4 & $25 \cdot 0-71.4$ & 57.5 & $50 \cdot 0$ & $16 \cdot 0-115 \cdot 1$ & $60 \cdot 4$ & $50 \cdot 0$ & $32 \cdot 1-64 \cdot 3$ & 57.9 & $64 \cdot 3$ & $35 \cdot 7-142 \cdot 9$ \\
\hline Hard margarine (brick) (g) & $26 \cdot 3$ & 1.4 & $0.7-3.1$ & 44.1 & 8.6 & $2 \cdot 9-17.9$ & 61.9 & 4.7 & $2 \cdot 9-7 \cdot 1$ & 66.8 & 5.4 & $2 \cdot 9-10.2$ \\
\hline $\begin{array}{l}\text { Starchy vegetables (e.g. potato, sweet potato, } \\
\text { mealies) with fat added (e.g. potato chips) (g) }\end{array}$ & $22 \cdot 0$ & $17 \cdot 1$ & $8 \cdot 6-17.9$ & $72 \cdot 0$ & 33.6 & $10 \cdot 0-64 \cdot 3$ & $34 \cdot 2$ & $13 \cdot 4$ & $8 \cdot 6-23 \cdot 6$ & 83.7 & $52 \cdot 9$ & $25 \cdot 7-107 \cdot 1$ \\
\hline $\begin{array}{l}\text { Beans, cooked (e.g. haricot, sugar beans, canned } \\
\text { baked beans) (g) }\end{array}$ & 21.5 & $14 \cdot 1$ & $7 \cdot 4-23 \cdot 7$ & $59 \cdot 7$ & 14.9 & $7 \cdot 7-33 \cdot 6$ & 39.1 & 8.6 & $2 \cdot 9-20 \cdot 0$ & $66 \cdot 8$ & 14.3 & $6 \cdot 4-35 \cdot 7$ \\
\hline $\begin{array}{l}\text { Orange/yellow/green coloured flesh fruit (e.g. } \\
\text { mango) (g) }\end{array}$ & $15 \cdot 6$ & $17 \cdot 1$ & $6 \cdot 4-34 \cdot 3$ & $29 \cdot 6$ & $37 \cdot 1$ & $8 \cdot 3-100 \cdot 0$ & 63.9 & $25 \cdot 7$ & $14 \cdot 6-61 \cdot 4$ & $55 \cdot 0$ & $54 \cdot 0$ & $22 \cdot 9-114 \cdot 3$ \\
\hline Nuts and seeds (mainly peanuts) $(\mathrm{g})$ & 14.5 & 11.4 & $5 \cdot 7-22 \cdot 9$ & 24.2 & $6 \cdot 3$ & $3 \cdot 7-15 \cdot 0$ & 54.5 & 5.7 & $3 \cdot 1-10 \cdot 0$ & 43.1 & $10 \cdot 0$ & $5 \cdot 0-20 \cdot 0$ \\
\hline $\begin{array}{l}\text { Other savoury liquids (gravy, packet soups, sauces, } \\
\text { etc.) (g) }\end{array}$ & 14.5 & $5 \cdot 7$ & $3 \cdot 4-10 \cdot 3$ & $32 \cdot 3$ & 8.6 & $3 \cdot 8-27 \cdot 6$ & $31 \cdot 2$ & $8 \cdot 6$ & $5 \cdot 3-17 \cdot 1$ & 43.1 & $17 \cdot 1$ & $7 \cdot 6-30 \cdot 0$ \\
\hline Jam, syrup, honey, sugar-based sweets (no fat) (g) & $12 \cdot 4$ & $2 \cdot 0$ & $1 \cdot 0-4 \cdot 0$ & 38.2 & 4.0 & $1.4-10 \cdot 0$ & $55 \cdot 4$ & 3.8 & $2 \cdot 8-8 \cdot 6$ & $66 \cdot 3$ & 11.1 & $5 \cdot 0-25 \cdot 0$ \\
\hline Mahewe (non-alcoholic drink) (g) & $11 \cdot 3$ & $100 \cdot 0$ & $71.4-350 \cdot 0$ & 20.4 & 31.3 & $17 \cdot 9-71 \cdot 4$ & 33.2 & 71.4 & $35 \cdot 7-71 \cdot 4$ & 47.5 & 71.4 & $35 \cdot 7-142 \cdot 9$ \\
\hline Venison, goat, rabbit, cooked (g) & 9.1 & 8.6 & $3 \cdot 1-14 \cdot 3$ & 30.1 & $17 \cdot 1$ & $8 \cdot 6-44 \cdot 2$ & 8.9 & 6.5 & $3 \cdot 6-12 \cdot 9$ & 8.4 & 5.4 & $2 \cdot 1-23 \cdot 1$ \\
\hline Soyabean products (e.g. Toppers) (g) & $8 \cdot 6$ & 11.4 & $5 \cdot 7-15 \cdot 4$ & 37.1 & 1.9 & $0.7-6 \cdot 3$ & 25.7 & $12 \cdot 1$ & $8 \cdot 6-24 \cdot 3$ & 43.6 & 7.4 & $1 \cdot 8-20 \cdot 3$ \\
\hline $\operatorname{Atchar}(\mathrm{g})$ & 5.9 & 2.9 & $1 \cdot 4-7 \cdot 0$ & $7 \cdot 0$ & 0.9 & $0.4-3.6$ & 47.5 & $6 \cdot 2$ & $1 \cdot 4-17 \cdot 1$ & 32.2 & $2 \cdot 4$ & $1 \cdot 3-17.3$ \\
\hline
\end{tabular}




\begin{tabular}{|c|c|c|c|c|c|c|c|c|c|c|c|c|}
\hline & \multicolumn{6}{|c|}{ Rural men ( $n$ 186) } & \multicolumn{6}{|c|}{ Urban men ( $n$ 202) } \\
\hline & \multicolumn{3}{|c|}{2005} & \multicolumn{3}{|c|}{2010} & \multicolumn{3}{|c|}{2005} & \multicolumn{3}{|c|}{2010} \\
\hline & $\%^{*}$ & Median & P25-P75 & $\%^{*}$ & Median & P25-P75 & $\%^{*}$ & Median & P25-P75 & $\%^{*}$ & Median & P25-P75 \\
\hline Salad dressings, mayonnaise, sandwich spread (g) & 4.3 & $2 \cdot 3$ & $1.0-4 \cdot 2$ & $29 \cdot 0$ & 7.6 & $2 \cdot 1-14 \cdot 0$ & $45 \cdot 0$ & 4.3 & $2 \cdot 9-10 \cdot 3$ & $42 \cdot 6$ & 8.8 & $4 \cdot 3-32 \cdot 6$ \\
\hline Tomato sauce $(\mathrm{g})$ & 3.8 & $16 \cdot 0$ & $7 \cdot 1-32 \cdot 9$ & 34.4 & $2 \cdot 9$ & $1.4-7.9$ & 53.5 & 4.3 & $2 \cdot 9-8.6$ & 58.4 & 5.6 & $2 \cdot 9-12 \cdot 9$ \\
\hline Mutton, cooked (g) & 3.8 & $3 \cdot 1$ & $2 \cdot 1-9 \cdot 7$ & 28.5 & 8.6 & $4 \cdot 3-21 \cdot 4$ & 18.8 & 11.5 & $3 \cdot 6-15 \cdot 7$ & 36.6 & 8.6 & $4 \cdot 3-21 \cdot 4$ \\
\hline Raw salads $(\mathrm{g})$ & 3.8 & $2 \cdot 1$ & $1.0-8 \cdot 6$ & 7.5 & 3.5 & $1.5-7.0$ & 34.2 & 8.6 & $4 \cdot 3-12 \cdot 9$ & 33.2 & 8.6 & $4 \cdot 0-17 \cdot 1$ \\
\hline Maas/sour milk (g) & 3.8 & 51.4 & $8 \cdot 6-180 \cdot 0$ & 11.3 & 86.6 & $51.4-171.4$ & $25 . \overline{7}$ & 30.0 & $16 \cdot 9-57 \cdot 1$ & $37 \cdot 1$ & 28.6 & $0.4-57.1$ \\
\hline Mixed dishes like bean soup, biryani (g) & 3.8 & $32 \cdot 1$ & $26 \cdot 7-89 \cdot 1$ & $4 \cdot 3$ & 11.3 & $6 \cdot 5-44 \cdot 3$ & $22 \cdot 8$ & $15 \cdot 0$ & $10 \cdot 0-22 \cdot 9$ & $12 \cdot 9$ & 28.6 & $10 \cdot 7-44 \cdot 6$ \\
\hline Soft margarine (tub) (g) & $2 \cdot 2$ & 1.2 & $0.6-1.4$ & 4.8 & 3.2 & $1 \cdot 4-5 \cdot 7$ & 7.4 & $5 \cdot 0$ & $2.9-10.0$ & $24 \cdot 3$ & 1.4 & $0.4-7.0$ \\
\hline Squash, cold drink (g) & 1.6 & 85.7 & $64 \cdot 3-96 \cdot 4$ & 27.4 & 42.9 & $16 \cdot 1-142 \cdot 9$ & $27 \cdot 2$ & 35.7 & $16 \cdot 0-71 \cdot 4$ & 35.6 & 71.4 & $33 \cdot 9-185 \cdot 7$ \\
\hline Sweet cookies, biscuits, rusks (g) & 1.6 & 5.7 & $5 \cdot 7-14 \cdot 3$ & 14.5 & $2 \cdot 1$ & $1 \cdot 1-8.6$ & $32 . \overline{7}$ & $5 \cdot 7$ & $2 \cdot 1-19 \cdot 3$ & $27 \cdot 7$ & 11.4 & $4.9-24 \cdot 3$ \\
\hline $\begin{array}{l}\text { Low-calorie cold drinks (carbonated \& ready-to-mix } \\
\text { types) (g) }\end{array}$ & 1.6 & $42 \cdot 9$ & $21.4-220.0$ & $2 \cdot 7$ & $100 \cdot 0$ & $96 \cdot 4-178 \cdot 6$ & 11.9 & $60 \cdot 7$ & $32 \cdot 1-95 \cdot 4$ & $26 \cdot 2$ & $128 \cdot 6$ & $50 \cdot 0-214 \cdot 3$ \\
\hline Dairy-fruit mix (g) & 1.6 & $32 \cdot 1$ & $16 \cdot 0-150 \cdot 0$ & $3 \cdot 2$ & $21 \cdot 0$ & $16 \cdot 1-500 \cdot 0$ & $20 \cdot 3$ & 35.7 & $28 \cdot 6-57 \cdot 1$ & 8.4 & $35 \cdot 7$ & $25 \cdot 7-128 \cdot 6$ \\
\hline Fruit juice (all types) (g) & 1.6 & $32 \cdot 1$ & $32 \cdot 1-71 \cdot 4$ & $1 \cdot 1$ & $82 \cdot 1$ & $21 \cdot 4-142.9$ & 13.9 & $42 \cdot 9$ & $10 \cdot 6-85 \cdot 7$ & $15 \cdot 3$ & $32 \cdot 1$ & $12 \cdot 6-71 \cdot 4$ \\
\hline Savoury snacks (e.g. potato crisps, niknaks, etc.) (g) & 1.1 & $5 \cdot 3$ & $3 \cdot 4-7 \cdot 1$ & 9.7 & $2 \cdot 9$ & $0.9-13.7$ & 31.7 & $7 \cdot 3$ & $3.4-10.9$ & $48 \cdot 0$ & 6.9 & $3 \cdot 3-10 \cdot 3$ \\
\hline Peanut butter (g) & 1.1 & 1.1 & $0.7-1 \cdot 4$ & $11 \cdot 3$ & 4.3 & $0.7-11.4$ & 29.7 & $2 \cdot 9$ & $1.4-4.6$ & 31.2 & $4 \cdot 3$ & $1.4-5.7$ \\
\hline Medium-fat fish, cooked (g) & 1.1 & 3.9 & $1.7-6.0$ & 0.5 & $2 \cdot 9$ & $2.9-2.9$ & $27 \cdot 2$ & 5.7 & $4 \cdot 3-11 \cdot 4$ & 11.4 & 4.7 & $2 \cdot 9-8 \cdot 6$ \\
\hline Beef, stewed with potato and/or vegetables (g) & 1.1 & $15 \cdot 7$ & $15 \cdot 7-15 \cdot 7$ & 9.1 & $15 \cdot 7$ & $7 \cdot 9-31 \cdot 4$ & 0.5 & 31.4 & $31 \cdot 4-31.4$ & $22 \cdot 8$ & $22 \cdot 9$ & $8 \cdot 6-47 \cdot 1$ \\
\hline Fudge, toffee, caramel (g) & 1.1 & 4.3 & $3 \cdot 6-5 \cdot 0$ & 1.1 & $15 \cdot 2$ & $4 \cdot 3-26 \cdot 1$ & $22 \cdot 3$ & 1.4 & $0.7-3 \cdot 6$ & 8.4 & $2 \cdot 9$ & $0 \cdot 9-7 \cdot 1$ \\
\hline Horse, donkey, cooked (g) & 1.1 & 20.6 & $7 \cdot 7-33.4$ & 9.7 & $19 \cdot 3$ & $5 \cdot 9-29.0$ & 0.0 & & 0 & 1.0 & 4.3 & $0.7-7.9$ \\
\hline $\begin{array}{l}\text { Milk products, made with full-fat milk (e.g. custard, } \\
\text { beverages, melkkos, etc.) (g) }\end{array}$ & 0.5 & 3.6 & $3 \cdot 6-3 \cdot 6$ & $11 \cdot 3$ & 1.4 & $0.9-3.6$ & $23 \cdot 8$ & $2 \cdot 9$ & $1 \cdot 7-7 \cdot 0$ & 39.6 & 3.6 & $1 \cdot 4-7 \cdot 1$ \\
\hline $\begin{array}{l}\text { Baked sweet foods (e.g. cake, pudding, tart, } \\
\text { muffins, scones) (g) }\end{array}$ & 0.5 & $5 \cdot 7$ & $5 \cdot 7-5 \cdot 7$ & $17 \cdot 2$ & $6 \cdot 1$ & $3 \cdot 1-22 \cdot 9$ & $12 \cdot 9$ & $5 \cdot 1$ & $2 \cdot 9-13 \cdot 3$ & 31.7 & $10 \cdot 7$ & $4 \cdot 6-22 \cdot 8$ \\
\hline Chocolate and chocolate-based sweets (g) & 0.5 & 2.6 & $2 \cdot 6-2 \cdot 6$ & 8.6 & 3.7 & $2 \cdot 1-20 \cdot 0$ & 18.8 & 1.6 & $0.6-3.9$ & 21.8 & 4.2 & $1.6-8.6$ \\
\hline Dried fruit $(\mathrm{g})$ & 0.5 & 1.0 & $1.0-1 \cdot 0$ & 8.6 & 4.6 & $2 \cdot 8-13 \cdot 2$ & 9.9 & 1.4 & $0.9-5 \cdot 0$ & 20.8 & 12.5 & $5 \cdot 4-25 \cdot 7$ \\
\hline Cheese, Cheddar (g) & 0.5 & 1.4 & $1.4-1.4$ & 0.0 & & 0 & $24 \cdot 3$ & 1.4 & $1 \cdot 1-2 \cdot 9$ & 11.4 & 1.4 & $1 \cdot 0-3 \cdot 1$ \\
\hline Pork, cooked $(\mathrm{g})$ & 0.5 & $3 \cdot 1$ & $3 \cdot 1-3 \cdot 1$ & 5.9 & $12 \cdot 9$ & $7 \cdot 1-21 \cdot 4$ & 0.5 & $42 \cdot 9$ & $42 \cdot 9-42 \cdot 9$ & 6.9 & $16 \cdot 1$ & $5 \cdot 4-25 \cdot 7$ \\
\hline $\begin{array}{l}\text { Savoury dishes (e.g. macaroni cheese, savoury tart, } \\
\text { pizza, samosa, spaghetti bolognaise, pies) (g) }\end{array}$ & 0.0 & & 0 & 3.2 & 4.6 & $2 \cdot 9-8 \cdot 6$ & 29.7 & $17 \cdot 1$ & $5 \cdot 0-35.6$ & $25 \cdot 2$ & $10 \cdot 0$ & $5 \cdot 0-31 \cdot 3$ \\
\hline Ice cream, all types (g) & 0.0 & & 0 & $2 \cdot 7$ & 8.0 & $5 \cdot 4-8 \cdot 0$ & 19.8 & 8.6 & $3.4-28 \cdot 6$ & $30 \cdot 2$ & $10 \cdot 7$ & $5 \cdot 4-27 \cdot 9$ \\
\hline Low-fat fish, cooked (g) & 0.0 & & 0 & $2 \cdot 7$ & $2 \cdot 9$ & $2 \cdot 1-5 \cdot 7$ & 0.5 & $9 \cdot 3$ & $9 \cdot 3-9 \cdot 3$ & 43.6 & 9.3 & $4 \cdot 7-18 \cdot 6$ \\
\hline Canned fruit/dried stewed with sugar $(\mathrm{g})$ & 0.0 & & 0 & 1.6 & $15 \cdot 7$ & $3 \cdot 6-107 \cdot 1$ & 19.8 & $10 \cdot 7$ & $4 \cdot 6-18 \cdot 6$ & 23.8 & $10 \cdot 1$ & $6 \cdot 8-21 \cdot 4$ \\
\hline Red-flesh fruit (e.g. pomegranate) (g) & 0.0 & & 0 & $17 \cdot 7$ & $14 \cdot 3$ & $7 \cdot 6-34.3$ & 1.5 & 7.9 & $4 \cdot 3-94 \cdot 3$ & 20.8 & $17 \cdot 1$ & $6 \cdot 1-31 \cdot 4$ \\
\hline Spirits (e.g. gin, brandy, whiskey, etc.) (g) & 0.0 & & 0 & 7.5 & $2 \cdot 8$ & $1 \cdot 1-5 \cdot 4$ & 3.0 & 4.4 & $1 \cdot 7-6 \cdot 4$ & $12 \cdot 9$ & $9 \cdot 9$ & $4 \cdot 3-28 \cdot 6$ \\
\hline Yoghurt, drinking, low-fat $(\mathrm{g})$ & 0.0 & & 0 & 2.7 & 6.3 & $5.4-17.9$ & 5.9 & $14 \cdot 3$ & $7 \cdot 9-25 \cdot 9$ & 13.9 & $19 \cdot 3$ & $7 \cdot 2-39 \cdot 3$ \\
\hline Breakfast cereals (e.g. corn flakes) (g) & 0.0 & & 0 & 1.6 & 8.3 & $1.9-10.7$ & 8.9 & 14.3 & $5.4-21 \cdot 4$ & 11.9 & $16 \cdot 1$ & $4.6-50.0$ \\
\hline Yoghurt, fruit, low-fat (g) & 0.0 & & 0 & $2 \cdot 2$ & 6.7 & $5 \cdot 4-39.7$ & $10 \cdot 9$ & 26.8 & $12 \cdot 4-50 \cdot 0$ & 8.4 & 21.4 & $7 \cdot 1-71 \cdot 4$ \\
\hline Animal fat (e.g. beef, mutton, chicken) (g) & 0.0 & & 0 & $2 \cdot 2$ & 1.3 & $0.7-4 \cdot 4$ & 0.0 & & 0 & $12 \cdot 9$ & 2.5 & $1 \cdot 0-7 \cdot 0$ \\
\hline Yoghurt, low-fat, plain (g) & 0.0 & & 0 & 0.5 & $10 \cdot 7$ & $10 \cdot 7-10 \cdot 7$ & 5.9 & 17.9 & $14 \cdot 3-28 \cdot 6$ & 4.5 & $10 \cdot 7$ & $9 \cdot 0-25 \cdot 7$ \\
\hline $\begin{array}{l}\text { Fish products (e.g. fish cakes, fingers, casserole, } \\
\text { salad) (g) }\end{array}$ & 0.0 & & 0 & $1 \cdot 1$ & $6 \cdot 4$ & $4 \cdot 3-8 \cdot 6$ & 7.9 & 3.6 & $1.8-11.8$ & 1.0 & $52 \cdot 9$ & $20 \cdot 0-85 \cdot 7$ \\
\hline
\end{tabular}

PURE-NWP-SA, Prospective Urban and Rural Epidemiology in the North West Province of South Africa; P25, 25th percentile; P75, 75th percentile; TE, total energy.

*Percentage of consumers. 


\begin{tabular}{|c|c|c|c|c|c|c|c|c|c|c|c|c|}
\hline & \multicolumn{6}{|c|}{ Rural women $(n 411)$} & \multicolumn{6}{|c|}{ Urban women $(n$ 355) } \\
\hline & \multicolumn{3}{|c|}{2005} & \multicolumn{3}{|c|}{2010} & \multicolumn{3}{|c|}{2005} & \multicolumn{3}{|c|}{2010} \\
\hline & $\%^{*}$ & Median & P25-P75 & $\%^{*}$ & Median & P25-P75 & $\%^{*}$ & Median & P25-P75 & $\%^{*}$ & Median & P25-P75 \\
\hline Energy intake (MJ) & - & $6 \cdot 2$ & $5 \cdot 0-7 \cdot 6$ & - & 9.1 & $6 \cdot 9-12 \cdot 8$ & - & $9 \cdot 0$ & $6.5-11.6$ & - & $11 \cdot 7$ & $8.9-14.9$ \\
\hline$\%$ of TE from protein & - & 10.9 & $9.9-12.0$ & - & 11.1 & $9 \cdot 6-12 \cdot 9$ & - & 12.5 & $11 \cdot 3-13.5$ & - & 12.5 & $11 \cdot 1-14 \cdot 3$ \\
\hline$\%$ of TE from total fat & - & $20 \cdot 3$ & $15 \cdot 6-24.5$ & - & 22.9 & $17 \cdot 7-30 \cdot 0$ & - & 28.4 & $23.7-31.9$ & - & $27 \cdot 7$ & $22 \cdot 8-32 \cdot 3$ \\
\hline$\%$ of TE from total carbohydrate & - & 66.7 & $61.3-71.9$ & - & 61.7 & $53.8-67.7$ & - & 55.7 & $51 \cdot 1-60 \cdot 2$ & - & 54.1 & $49.5-59.8$ \\
\hline $\begin{array}{l}\text { Cooked porridge (e.g. maize meal, maltabella, } \\
\text { oats) (g) }\end{array}$ & $100 \cdot 0$ & $700 \cdot 0$ & $500 \cdot 0-850 \cdot 0$ & $100 \cdot 0$ & $600 \cdot 0$ & $314 \cdot 3-900 \cdot 0$ & $99 \cdot 4$ & $405 \cdot 3$ & $294 \cdot 6-700 \cdot 0$ & $100 \cdot 0$ & $431 \cdot 1$ & $250 \cdot 0-675 \cdot 0$ \\
\hline $\begin{array}{l}\text { Samp, maize rice, wheat rice, rice, macaroni, } \\
\text { spaghetti (g) }\end{array}$ & $98 \cdot 8$ & $83 \cdot 4$ & $44 \cdot 6-125 \cdot 0$ & $98 \cdot 1$ & 71.4 & $40 \cdot 3-125 \cdot 0$ & $97 \cdot 2$ & $71 \cdot 4$ & $40 \cdot 1-125 \cdot 7$ & 98.9 & $79 \cdot 7$ & $47 \cdot 1-140 \cdot 3$ \\
\hline Sunflower oil $(g)$ & 97.8 & $5 \cdot 1$ & $2 \cdot 6-8 \cdot 6$ & $95 \cdot 6$ & $3 \cdot 0$ & $1 \cdot 4-6 \cdot 0$ & $99 \cdot 2$ & $9 \cdot 1$ & $5 \cdot 9-12 \cdot 1$ & $97 \cdot 7$ & 3.7 & $2 \cdot 0-6 \cdot 4$ \\
\hline Sugar, white and brown (g) & $97 \cdot 3$ & $19 \cdot 0$ & $12 \cdot 0-30 \cdot 6$ & $96 \cdot 6$ & 33.3 & $14.4-63.0$ & 99.7 & $24 \cdot 6$ & $13.9-41.6$ & $98 \cdot 3$ & $30 \cdot 7$ & $14 \cdot 1-56 \cdot 2$ \\
\hline $\begin{array}{l}\text { Green vegetables (e.g. spinach, cabbage, } \\
\text { coleslaw, broccoli) (g) }\end{array}$ & 95.9 & $17 \cdot 1$ & $9 \cdot 3-26 \cdot 4$ & 87.8 & $15 \cdot 8$ & $6 \cdot 6-28 \cdot 2$ & 98.9 & $18 \cdot 7$ & $11 \cdot 4-28 \cdot 6$ & 94.6 & $19 \cdot 6$ & $9 \cdot 0-36 \cdot 2$ \\
\hline Bread, rolls, vetkoek (g) & 95.6 & 71.4 & $42 \cdot 9-114 \cdot 3$ & $96 \cdot 4$ & $67 \cdot 4$ & $33 \cdot 9-129 \cdot 6$ & 98.0 & 97.5 & $59 \cdot 1-153 \cdot 9$ & $97 \cdot 7$ & 94.3 & $51 \cdot 4-161 \cdot 4$ \\
\hline $\begin{array}{l}\text { Chicken, turkey, goose, duck, cooked, including } \\
\text { Kentucky (g) }\end{array}$ & $94 \cdot 2$ & 14.7 & $7 \cdot 7-25 \cdot 7$ & 94.6 & $20 \cdot 9$ & $9 \cdot 3-41 \cdot 9$ & 96.9 & 31.9 & $18 \cdot 6-46 \cdot 4$ & $97 \cdot 7$ & 45.4 & $26 \cdot 9-78 \cdot 3$ \\
\hline $\begin{array}{l}\text { Yellow and red vegetables (e.g. red peppers, } \\
\text { carrots, pumpkin, beetroot) (q) }\end{array}$ & 93.4 & $19 \cdot 3$ & $11 \cdot 4-25 \cdot 3$ & $92 \cdot 9$ & $22 \cdot 4$ & $8 \cdot 5-47 \cdot 6$ & $94 \cdot 9$ & $27 \cdot 0$ & $17 \cdot 9-44 \cdot 4$ & $98 \cdot 0$ & $32 \cdot 1$ & $19.7-57.5$ \\
\hline $\begin{array}{l}\text { Other cooked vegetables (e.g. parsnips, green } \\
\text { beans, green peas) (g) }\end{array}$ & $92 \cdot 2$ & $4 \cdot 3$ & $2 \cdot 1-7 \cdot 4$ & 91.5 & $7 \cdot 0$ & $3 \cdot 0-18 \cdot 1$ & $98 \cdot 0$ & $9 \cdot 7$ & $5 \cdot 1-16 \cdot 4$ & 98.0 & $24 \cdot 0$ & $12 \cdot 0-44 \cdot 7$ \\
\hline Coffee and tea $(\mathrm{g})$ & $91 \cdot 7$ & $300 \cdot 0$ & $300 \cdot 0-428.6$ & $93 \cdot 2$ & $600 \cdot 0$ & $300 \cdot 0-880 \cdot 0$ & 95.5 & 257.1 & $171 \cdot 4-440 \cdot 0$ & 94.6 & 440.0 & $227 \cdot 9-660 \cdot 0$ \\
\hline Tomato, cooked and raw (g) & 88.1 & $5 \cdot 1$ & $3 \cdot 4-9 \cdot 1$ & 84.4 & 4.8 & $1.9-10.6$ & 98.0 & 11.6 & $6 \cdot 9-21 \cdot 4$ & 92.7 & 12.2 & $5.2-23.5$ \\
\hline White-flesh fruit (e.g. apples, bananas, pears) (g) & $77 \cdot 6$ & $25 \cdot 1$ & $15 \cdot 4-43 \cdot 6$ & $88 \cdot 3$ & $55 \cdot 0$ & $22 \cdot 9-127 \cdot 1$ & $84 \cdot 2$ & 65.0 & $31.4-92.9$ & $96 \cdot 6$ & 100.0 & $60 \cdot 0-181 \cdot 0$ \\
\hline $\begin{array}{l}\text { Starchy vegetables (e.g. potato, sweet potato, } \\
\text { mealies) }(\mathrm{g})\end{array}$ & $71 \cdot 0$ & 11.4 & $6.9-21 \cdot 4$ & $88 \cdot 3$ & $25 \cdot 0$ & $10 \cdot 0-60 \cdot 7$ & 97.7 & 23.6 & $11 \cdot 0-52 \cdot 6$ & $95 \cdot 5$ & $44 \cdot 3$ & $20 \cdot 0-80 \cdot 6$ \\
\hline $\begin{array}{l}\text { Canned fish (e.g. pilchards in tomato sauce, } \\
\text { sardines, tuna) (g) }\end{array}$ & $69 \cdot 3$ & 6.0 & $3 \cdot 0-12 \cdot 0$ & $51 \cdot 3$ & 4.3 & $1 \cdot 6-12 \cdot 0$ & 63.9 & $6 \cdot 3$ & $3 \cdot 0-13 \cdot 3$ & $50 \cdot 7$ & 6.0 & $3 \cdot 0-16 \cdot 6$ \\
\hline Beef, cooked + meatballs $(\mathrm{g})$ & 59.4 & 9.6 & $3 \cdot 4-14 \cdot 3$ & 58.4 & $10 \cdot 7$ & $5 \cdot 4-25 \cdot 0$ & 85.9 & $24 \cdot 3$ & $14 \cdot 3-40 \cdot 1$ & 73.5 & $22 \cdot 7$ & $11 \cdot 1-51.4$ \\
\hline Milk, full-fat (g) & 49.4 & 53.6 & $28 \cdot 6-120$ & $75 \cdot 2$ & 140.7 & $60 \cdot 0-235.7$ & 90.1 & $100 \cdot 0$ & $62 \cdot 1-164 \cdot 3$ & 87.6 & 139.3 & $72 \cdot 9-220 \cdot 0$ \\
\hline Non-dairy creamer, milk blend (g) & 48.7 & 6.0 & $4.0-10.4$ & 49.9 & 9.0 & $4 \cdot 7-24 \cdot 0$ & $17 \cdot 2$ & 5.1 & $2 \cdot 0-12 \cdot 0$ & $14 \cdot 1$ & 9.6 & $4 \cdot 3-22 \cdot 9$ \\
\hline $\begin{array}{l}\text { Organ meats (e.g. liver, kidney, tripe, offal, heart, } \\
\text { giblets, lung, tongue, etc.) (g) }\end{array}$ & $47 \cdot 4$ & 8.9 & $5 \cdot 7-17 \cdot 1$ & $62 \cdot 0$ & 8.6 & $4 \cdot 3-20 \cdot 0$ & $80 \cdot 3$ & $17 \cdot 1$ & $8 \cdot 6-27 \cdot 4$ & $76 \cdot 9$ & 23.1 & $10 \cdot 7-44 \cdot 4$ \\
\hline Eggs (g) & 45.5 & 14.9 & $10 \cdot 7-29 \cdot 7$ & $58 \cdot 2$ & $16 \cdot 7$ & $7 \cdot 1-42 \cdot 9$ & $70 \cdot 4$ & $22 \cdot 3$ & $14 \cdot 9-36 \cdot 9$ & $79 \cdot 7$ & 28.6 & $14 \cdot 3-44 \cdot 0$ \\
\hline Carbonated cold drinks (g) & 37.0 & $37 \cdot 4$ & $25 \cdot 0-61 \cdot 6$ & 63.0 & $26 \cdot 9$ & $12 \cdot 6-96 \cdot 4$ & 65.1 & $42 \cdot 9$ & $32 \cdot 1-71 \cdot 4$ & $51 \cdot 3$ & 71.4 & $37 \cdot 6-150 \cdot 0$ \\
\hline $\begin{array}{l}\text { Beans, cooked (e.g. haricot, sugar beans, canned } \\
\text { baked beans) (g) }\end{array}$ & 34.8 & 11.9 & $6 \cdot 0-20 \cdot 0$ & $61 \cdot 6$ & $19 \cdot 1$ & $8 \cdot 3-42 \cdot 9$ & $48 \cdot 2$ & $5 \cdot 7$ & $2.9-11.9$ & 78.9 & $16 \cdot 4$ & $6 \cdot 5-32 \cdot 6$ \\
\hline $\begin{array}{l}\text { Meat products (e.g. boerewors, biltong, ham, } \\
\text { corned meat, viennas, frankfurter, salami, } \\
\text { patties) (g) }\end{array}$ & $30 \cdot 4$ & $7 \cdot 7$ & $3.9-10 \cdot 6$ & 68.9 & 8.6 & $4 \cdot 3-22 \cdot 9$ & $92 \cdot 4$ & $16 \cdot 3$ & $9 \cdot 6-27 \cdot 9$ & $85 \cdot 9$ & $20 \cdot 0$ & $9 \cdot 0-42 \cdot 9$ \\
\hline $\begin{array}{l}\text { Starchy vegetables (e.g. potato, sweet potato, } \\
\text { mealies) with fat added (e.g. potato chips) (g) }\end{array}$ & $24 \cdot 8$ & $17 \cdot 1$ & $8 \cdot 9-26 \cdot 7$ & $77 \cdot 1$ & $34 \cdot 3$ & $12 \cdot 9-68 \cdot 6$ & $38 \cdot 6$ & $16 \cdot 0$ & $8 \cdot 6-22 \cdot 9$ & 89.6 & 48.6 & $20 \cdot 4-87 \cdot 0$ \\
\hline Beer, commercial and homemade $(\mathrm{g})$ & $24 \cdot 3$ & $496 \cdot 4$ & $125 \cdot 0-1985 \cdot 7$ & $23 \cdot 1$ & $142 \cdot 9$ & $60 \cdot 0-500 \cdot 0$ & $42 \cdot 5$ & 571.4 & $214 \cdot 3-1050 \cdot 0$ & 37.5 & $400 \cdot 0$ & $142 \cdot 9-857 \cdot 1$ \\
\hline Hard margarine (brick) (g) & $22 \cdot 6$ & & $0.7-4 \cdot 3$ & 53.0 & $5 \cdot 4$ & $2 \cdot 1-12 \cdot 9$ & 73.5 & $5 \cdot 0$ & $2 \cdot 9-8.6$ & 64.5 & 5.5 & $1.8-10 \cdot 0$ \\
\hline Jam, syrup, honey, sugar-based sweets (no fat) (g) & $20 \cdot 4$ & 1.4 & $1.0-4.1$ & 50.9 & $5 \cdot 0$ & $2 \cdot 1-14 \cdot 0$ & 67.6 & $4 \cdot 3$ & $2 \cdot 6-8 \cdot 8$ & 75.8 & $13 \cdot 3$ & $5 \cdot 0-29 \cdot 4$ \\
\hline $\begin{array}{l}\text { Other savoury liquids (gravy, packet soups, sauces, } \\
\text { etc.) }(\mathrm{g})\end{array}$ & $18 \cdot 0$ & 5.7 & $3 \cdot 4-10 \cdot 3$ & 39.7 & $12 \cdot 9$ & $4 \cdot 3-35 \cdot 7$ & $35 \cdot 8$ & 8.6 & $4 \cdot 3-17 \cdot 1$ & 38.0 & $13 \cdot 6$ & $5 \cdot 7-25 \cdot 7$ \\
\hline $\begin{array}{l}\text { Orange/yellow/green coloured flesh fruit (e.g. } \\
\text { mango) (g) }\end{array}$ & 17.5 & $12 \cdot 9$ & $6 \cdot 4-25 \cdot 7$ & $28 \cdot 2$ & 31.4 & $15 \cdot 0-94 \cdot 3$ & $62 \cdot 0$ & 31.4 & $15 \cdot 7-71 \cdot 8$ & $53 \cdot 0$ & 67.9 & $32 \cdot 5-164 \cdot 3$ \\
\hline Nuts and seeds $(\mathrm{g})$ & 17.5 & 5.7 & $2 \cdot 9-11.4$ & $25 \cdot 3$ & $5 \cdot 0$ & $2 \cdot 6-10 \cdot 0$ & 48.5 & $5 \cdot 0$ & $2 \cdot 9-10 \cdot 0$ & $37 \cdot 7$ & $5 \cdot 0$ & $2 \cdot 6-11 \cdot 4$ \\
\hline Mahewe (non-alcoholic drink) (g) & $16 \cdot 8$ & $71 \cdot 4$ & $53 \cdot 6-350 \cdot 0$ & 23.4 & 53.6 & $17 \cdot 9-142 \cdot 9$ & $36 \cdot 1$ & $57 \cdot 1$ & $21.4-142 \cdot 9$ & 31.5 & 71.4 & $33.6-142.9$ \\
\hline Soyabean products $(\mathrm{g})$ & 13.4 & 11.4 & $7 \cdot 9-17 \cdot 1$ & $43 \cdot 1$ & $2 \cdot 6$ & $0.9-7.5$ & 33.5 & $12 \cdot 1$ & $5 \cdot 7-24 \cdot 3$ & $45 \cdot 9$ & $5 \cdot 7$ & $2 \cdot 6-17 \cdot 1$ \\
\hline Atchar $(\mathrm{g})$ & 12.4 & 7.0 & $3 \cdot 6-14 \cdot 3$ & 10.5 & 1.1 & $0.6-4.7$ & $57 \cdot 2$ & $7 \cdot 1$ & $2 \cdot 1-15 \cdot 0$ & 40.0 & $2 \cdot 3$ & $1 \cdot 1-7 \cdot 1$ \\
\hline
\end{tabular}




\begin{tabular}{|c|c|c|c|c|c|c|c|c|c|c|c|c|}
\hline & \multicolumn{6}{|c|}{ Rural women $(n 411)$} & \multicolumn{6}{|c|}{ Urban women ( $n$ 355) } \\
\hline & \multicolumn{3}{|c|}{2005} & \multicolumn{3}{|c|}{2010} & \multicolumn{3}{|c|}{2005} & \multicolumn{3}{|c|}{2010} \\
\hline & $\%^{*}$ & Median & P25-P75 & $\%^{*}$ & Median & P25-P75 & $\%^{*}$ & Median & P25-P75 & $\%^{*}$ & Median & P25-P75 \\
\hline Venison, goat, rabbit, cooked (g) & 8.5 & 6.4 & $3.6-9 \cdot 7$ & $22 \cdot 1$ & 8.6 & $4 \cdot 0-30 \cdot 4$ & 5.4 & 5.3 & $3.6-7.9$ & 3.7 & 5.4 & $3.3-8.6$ \\
\hline Tomato sauce $(\mathrm{g})$ & 7.8 & 8.0 & $4 \cdot 1-17 \cdot 9$ & $43 \cdot 6$ & 2.9 & $1.4-10.0$ & 54.6 & 3.9 & $2 \cdot 9-7 \cdot 1$ & 57.7 & $7 \cdot 1$ & $2 \cdot 9-14 \cdot 3$ \\
\hline Squash, cold drink (g) & 7.8 & 64.3 & $35.7-117.9$ & 37.0 & 46.4 & $17 \cdot 9-150 \cdot 0$ & 40.6 & 35.7 & $25 \cdot 4-65 \cdot 7$ & 35.8 & 87.6 & $42 \cdot 9-214 \cdot 3$ \\
\hline Mutton, cooked $(\mathrm{g})$ & $6 \cdot 1$ & 9.7 & $4 \cdot 9-14 \cdot 3$ & 32.6 & $6 \cdot 4$ & $3 \cdot 3-15.4$ & $22 \cdot 3$ & 9.7 & $3 \cdot 6-14 \cdot 3$ & 24.5 & 7.7 & $3.3-21.4$ \\
\hline Salad dressings, mayonnaise, sandwich spread (g) & 5.6 & 4.3 & $3.1-7.9$ & 48.7 & 6.0 & $2 \cdot 0-18 \cdot 6$ & $62 \cdot 8$ & $5 \cdot 0$ & $2 \cdot 9-13 \cdot 6$ & $56 \cdot 6$ & $5 \cdot 7$ & $2 \cdot 1-16$ \\
\hline Dairy-fruit mix (g) & 3.6 & 37.4 & $32.1-102.9$ & 3.9 & 23.2 & $13.4-42.9$ & $29 \cdot 3$ & $32 \cdot 1$ & $20.4-57.1$ & 11.3 & 47.4 & $28 \cdot 6-160 \cdot 7$ \\
\hline Sweet cookies, biscuits, rusks (g) & 3.4 & 3.1 & $2 \cdot 9-5 \cdot 3$ & 20.2 & 4.3 & $1.4-14.3$ & 52.4 & 6.9 & $2.9-13.9$ & $36 \cdot 3$ & $10 \cdot 7$ & $5 \cdot 4-25 \cdot 7$ \\
\hline Savoury snacks (e.g. potato crisps, niknaks, etc.) (g) & 2.9 & $6 \cdot 1$ & $4 \cdot 3-21 \cdot 8$ & 22.4 & 2.6 & $1 \cdot 1-6 \cdot 9$ & 50.7 & 6.9 & $3.4-12 \cdot 0$ & 59.7 & 6.9 & $3 \cdot 3-12 \cdot 0$ \\
\hline Raw salads $(\mathrm{g})$ & 2.9 & $8 \cdot 6$ & $2 \cdot 1-8 \cdot 6$ & 11.4 & 4.4 & $2 \cdot 3-9 \cdot 7$ & 44.5 & 8.6 & $4 \cdot 3-17.1$ & 43.7 & 8.6 & $3.3-18.0$ \\
\hline Mixed dishes like bean soup, biryani (g) & 2.9 & $20 \cdot 0$ & $10 \cdot 4-43.7$ & 3.6 & 9.7 & $2 \cdot 1-25 \cdot 7$ & 31.8 & $16 \cdot 4$ & $8 \cdot 6-31 \cdot 4$ & 14.4 & $10 \cdot 7$ & $7 \cdot 1-25 \cdot 7$ \\
\hline Peanut butter (g) & 2.7 & 1.4 & $0.7-2.9$ & 11.7 & 2.9 & $1 \cdot 4-4 \cdot 3$ & $49 \cdot 0$ & 2.9 & $1.4-5.0$ & 38.6 & 2.9 & $1 \cdot 4-5 \cdot 0$ \\
\hline Fruit juice (all types) (g) & $2 \cdot 2$ & 31.4 & $22 \cdot 9-35.7$ & 4.4 & 25.0 & $11.6-37.6$ & 14.6 & 32.1 & $17.9-57.1$ & 18.9 & 71.4 & $21 \cdot 4-150 \cdot 0$ \\
\hline Soft margarine (tub) (g) & 1.7 & 1.4 & $0.7-2.9$ & 9.0 & 6.4 & $2.9-14.9$ & 13.8 & $5 \cdot 0$ & $2.9-9.0$ & $36 \cdot 1$ & $2 \cdot 3$ & $0.8-7.3$ \\
\hline $\begin{array}{l}\text { Low-calorie cold drinks (carbonated and ready-to- } \\
\text { mix types) }(\mathrm{g} \text { ) }\end{array}$ & $1 \cdot 7$ & 35.7 & $16 \cdot 0-64 \cdot 3$ & 4.9 & $60 \cdot 7$ & $19 \cdot 6-100 \cdot 0$ & $20 \cdot 3$ & $39 \cdot 6$ & $29 \cdot 1-71 \cdot 4$ & 24.5 & 71.4 & $35 \cdot 7-128 \cdot 6$ \\
\hline Beef, stewed with potato and/or vegetables (g) & 1.7 & $15 \cdot 7$ & $7.9-31.4$ & 14.1 & 11.9 & $4.0-32.9$ & $2 \cdot 0$ & $18 \cdot 3$ & $7.9-31.4$ & $26 \cdot 2$ & $17 \cdot 1$ & $7 \cdot 9-47 \cdot 1$ \\
\hline Maas/sour milk (g) & 1.5 & 123.2 & $16 \cdot 0-180 \cdot 0$ & 10.5 & 77.1 & $21 \cdot 4-166 \cdot 1$ & 33.5 & 31.4 & $17.9-71.4$ & 41.1 & 19.4 & $0.7-44.7$ \\
\hline $\begin{array}{l}\text { Baked sweet foods (e.g. cake, pudding, tart, } \\
\text { muffins, scones) (g) }\end{array}$ & 1.2 & 20.0 & $15 \cdot 0-25 \cdot 0$ & 20.7 & 8.6 & $5 \cdot 0-21 \cdot 4$ & 25.9 & 4.6 & $2 \cdot 7-9 \cdot 2$ & 39.2 & 11.9 & $4 \cdot 3-27 \cdot 1$ \\
\hline Chocolate and chocolate-based sweets (g) & 1.2 & 2.1 & $0.7-6 \cdot 3$ & $16 \cdot 3$ & 3.6 & $1 \cdot 3-7 \cdot 1$ & 28.7 & $2 \cdot 0$ & $1 \cdot 0-4 \cdot 6$ & 30.7 & 5.0 & $2.4-8.6$ \\
\hline $\begin{array}{l}\text { Milk products, made with full-fat milk (e.g. custard, } \\
\text { beverages, melkkos, etc.) (g) }\end{array}$ & 0.7 & 3.6 & $3.6-8.9$ & $14 \cdot 1$ & $3 \cdot 2$ & $1 \cdot 1-4 \cdot 7$ & $40 \cdot 6$ & 3.0 & $1.4-6.9$ & $49 \cdot 3$ & 4.6 & $2 \cdot 9-12 \cdot 9$ \\
\hline Fudge, toffee, caramel (g) & 0.7 & $2 \cdot 1$ & $0.7-2 \cdot 9$ & 0.2 & 3.3 & $3 \cdot 3-3 \cdot 3$ & 33.8 & $2 \cdot 1$ & $1.4-3 \cdot 6$ & $11 \cdot 0$ & $4 \cdot 3$ & $1 \cdot 4-10 \cdot 7$ \\
\hline Yoghurt, drinking, low-fat (g) & 0.7 & 17.9 & $3.6-42.9$ & 2.9 & 17.9 & $7 \cdot 6-28.6$ & 14.4 & 25.0 & $12 \cdot 4-50 \cdot 0$ & 18.6 & $25 \cdot 0$ & $14 \cdot 3-35 \cdot 7$ \\
\hline Milk, low-fat $/ 2 \%(\mathrm{~g})$ & 0.7 & 60.0 & $35 \cdot 0-60 \cdot 0$ & 1.2 & 20.0 & $4.3-90.0$ & $2 \cdot 8$ & $60 \cdot 7$ & $8 \cdot 6-100 \cdot 0$ & $5 \cdot 6$ & $90 \cdot 7$ & $64 \cdot 3-227 \cdot 1$ \\
\hline Dried fruit $(\mathrm{g})$ & 0.5 & 21.9 & $8 \cdot 1-35.7$ & 8.8 & $6 \cdot 1$ & $4.6-22 \cdot 4$ & 17.5 & 2.9 & $1.0-5.6$ & 20.3 & $10 \cdot 7$ & $3.7-25.3$ \\
\hline Cheese, Cheddar (g) & 0.5 & 1.9 & $1.4-2 \cdot 4$ & 0.5 & 7.1 & $1.4-12.9$ & 30.7 & 1.4 & $1.1-2.9$ & $15 \cdot 8$ & 2.1 & $0.7-4 \cdot 3$ \\
\hline $\begin{array}{l}\text { Savoury dishes (e.g. macaroni cheese, savoury tart, } \\
\text { pizza, samosa, spaghetti bolognaise, pies) (g) }\end{array}$ & 0.2 & 53.6 & $53 \cdot 6-53 \cdot 6$ & 5.6 & $6 \cdot 4$ & $5 \cdot 0-12 \cdot 9$ & 38.6 & 11.7 & $5 \cdot 9-24 \cdot 3$ & 31.5 & $19 \cdot 3$ & $5 \cdot 0-34.9$ \\
\hline Medium-fat fish, cooked $(\mathrm{g})$ & 0.2 & $2 \cdot 9$ & $2 \cdot 9-2.9$ & 0.2 & 34.3 & $\begin{array}{r}34 \cdot 3-34 \cdot 3 \\
2\end{array}$ & $28 \cdot 7$ & $5 \cdot 7$ & $4 \cdot 6-11.4$ & $6 \cdot 8$ & $12 \cdot 7$ & $4 \cdot 7-20 \cdot 7$ \\
\hline $\begin{array}{l}\text { ICe cream, all types (g) } \\
\text { Canned fruit/dried stewed with sugar (q) }\end{array}$ & $\begin{array}{l}0.0 \\
0.0\end{array}$ & & 0 & $\begin{array}{l}1 \cdot 1 \\
5 \cdot 1\end{array}$ & $\begin{array}{l}5.4 \\
7.9\end{array}$ & $\begin{array}{l}2 \cdot 9-14 \cdot 3 \\
5 \cdot 4-19 \cdot 6\end{array}$ & $\begin{array}{l}28 \cdot 5 \\
29 \cdot 6\end{array}$ & $\begin{array}{l}10.7 \\
12.9\end{array}$ & $\begin{array}{l}3 \cdot 6-21 \cdot 4 \\
7 \cdot 4-20 \cdot 3\end{array}$ & $\begin{array}{l}41.4 \\
28.7\end{array}$ & $\begin{array}{l}15.4 \\
10.0\end{array}$ & $\begin{array}{l}5.4-21.9 \\
7.9-20.0\end{array}$ \\
\hline Low-fat fish, cooked $(\mathrm{g})$ & 0.0 & & 0 & 6.3 & $4 \cdot 3$ & $2.9-8.6$ & 1.4 & 4.7 & $2.9-11.4$ & 43.1 & 11.4 & $4.7-18.6$ \\
\hline Breakfast cereals (e.g. cornflakes) (g) & 0.0 & & 0 & 4.6 & 32.1 & $5.4-50.0$ & $16 \cdot 3$ & 8.6 & $5 \cdot 0-14 \cdot 3$ & 27.3 & $10 \cdot 7$ & $5 \cdot 7-25 \cdot 7$ \\
\hline Red-flesh fruit (e.g. pomegranate) (g) & 0.0 & & 0 & 11.9 & $15 \cdot 7$ & $7 \cdot 1-36 \cdot 4$ & 0.8 & 62.9 & $11.4-171.4$ & 24.8 & $24 \cdot 3$ & $8 \cdot 6-62 \cdot 9$ \\
\hline Yoghurt, fruit, low-fat (g) & 0.0 & & 0 & 6.8 & 11.8 & $5 \cdot 4-30 \cdot 4$ & $13 \cdot 2$ & 25.0 & $6.3-50.0$ & 14.6 & 21.4 & $7 \cdot 1-32 \cdot 9$ \\
\hline
\end{tabular}

PURE-NWP-SA, Prospective Urban and Rural Epidemiology in the North West Province of South Africa; P25, 25th percentile; P75, 75th percentile; TE, total energy. *Percentage of consumers. 


\begin{tabular}{|c|c|c|c|c|c|c|c|c|c|c|c|c|}
\hline & \multicolumn{6}{|c|}{ Rural men $(n 186)$} & \multicolumn{6}{|c|}{ Urban men ( $n$ 202) } \\
\hline & \multicolumn{3}{|c|}{2005} & \multicolumn{3}{|c|}{2010} & \multicolumn{3}{|c|}{2005} & \multicolumn{3}{|c|}{2010} \\
\hline & $\% *$ & Median & P25-P75 & $\%^{*}$ & Median & P25-P75 & $\%^{*}$ & Median & P25-P75 & $\%^{*}$ & Median & P25-P75 \\
\hline Cooked porridge (e.g. maize meal, oats) and maize-based drinks (\% of TE) & 99 & 35.9 & $24 \cdot 5-47 \cdot 8$ & 100 & $30 \cdot 0$ & $16 \cdot 5-45 \cdot 9$ & 100 & $26 \cdot 3$ & $19 \cdot 1-35 \cdot 7$ & 100 & 23.3 & $13 \cdot 2-34 \cdot 3$ \\
\hline $\begin{array}{l}\text { Starchy grains (cereals, bread, vetkoek), starchy vegetables (potato, sweet } \\
\text { potato, corn) (\% of TE) }\end{array}$ & 99 & 21.7 & $14 \cdot 7-33 \cdot 6$ & 100 & $19 \cdot 7$ & $11 \cdot 4-27 \cdot 4$ & 100 & $21 \cdot 1$ & $13 \cdot 6-28 \cdot 1$ & 100 & $20 \cdot 1$ & $15 \cdot 4-26 \cdot 6$ \\
\hline Fresh vegetables ( $\%$ of TE) & 99 & 1.3 & $0.6-2 \cdot 1$ & 98 & 0.9 & $0.4-2 \cdot 0$ & 100 & 1.5 & $1 \cdot 0-2 \cdot 2$ & 100 & 1.6 & $0.9-2 \cdot 6$ \\
\hline Fresh fruit and fruit juices & 69 & 1.0 & $0.5-1.8$ & 87 & $2 \cdot 4$ & $0.9-5 \cdot 0$ & 86 & $2 \cdot 1$ & $1 \cdot 1-3 \cdot 9$ & 96 & 3.6 & $1.8-6.4$ \\
\hline Legumes and legume products ( $\%$ of TE) & 29 & 1.1 & $0.6-2.5$ & 70 & 1.0 & $0.5-2.4$ & 53 & 0.8 & $0.4-1.7$ & 79 & 1.1 & $0.4-1.9$ \\
\hline Nuts and seeds (\% of TE) & 15 & 3.3 & $1.7-6 \cdot 8$ & 24 & $2 \cdot 0$ & $0.9-3.6$ & 54 & 1.4 & $0.9-2 \cdot 3$ & 43 & 1.6 & $0.8-3.6$ \\
\hline Milk and milk products ( $\%$ of TE) & 52 & $2 \cdot 3$ & $1 \cdot 3-5 \cdot 9$ & 84 & 3.8 & $1 \cdot 6-6 \cdot 2$ & 91 & 3.3 & $2 \cdot 1-5 \cdot 6$ & 94 & 3.9 & $2 \cdot 2-5 \cdot 8$ \\
\hline All meat, chicken, fish, and egg and products ( $\%$ of TE) & 99 & $6 \cdot 0$ & $3.7-8.9$ & 100 & $9 \cdot 7$ & $5 \cdot 1-14 \cdot 8$ & 100 & 13.3 & $9 \cdot 7-17 \cdot 6$ & 100 & $14 \cdot 0$ & $10 \cdot 1-18 \cdot 7$ \\
\hline All fats and oils and products (including ice cream) (\% of TE) & 99 & 4.0 & $2 \cdot 1-6 \cdot 2$ & 97 & $4 \cdot 2$ & $1.9-8.1$ & 100 & 6.3 & $4.4-8 \cdot 4$ & 100 & 4.2 & $2 \cdot 3-7.5$ \\
\hline $\begin{array}{l}\text { Sugar, syrups, sweets and refined products with sugar added (sweet cookies, } \\
\text { cakes, biscuits) (\% of TE) }\end{array}$ & 96 & 5.9 & $3 \cdot 4-8 \cdot 3$ & 99 & $9 \cdot 1$ & $4 \cdot 9-15 \cdot 0$ & 99 & $7 \cdot 1$ & $4 \cdot 7-10 \cdot 0$ & 100 & 8.6 & $4 \cdot 6-13.5$ \\
\hline Savoury snacks, dishes, sauces, seasoning and products (\% of TE) & 23 & 0.3 & $0.1-1 \cdot 1$ & 62 & 0.5 & $0.1-1 \cdot 1$ & 78 & 1.7 & $0.4-4.5$ & 87 & 1.9 & $0 \cdot 8-4 \cdot 1$ \\
\hline Alcoholic drinks (\% of TE) & 56 & $22 \cdot 7$ & $6 \cdot 4-41.8$ & 53 & $9 \cdot 4$ & $2.9-18.9$ & 70 & 11.5 & $5 \cdot 2-17 \cdot 4$ & 64 & $5 \cdot 8$ & $2 \cdot 4-15 \cdot 9$ \\
\hline
\end{tabular}

PURE-NWP-SA, Prospective Urban and Rural Epidemiology in the North West Province of South Africa; P25, 25th percentile; P75, 75th percentile; TE, total energy.

*Percentage of consumers.

Table 7 Percentage contribution of twelve food groups to total energy intake for the female participants according to rural/urban residence in 2005 and 2010 ; PURE-NWP-SA study

\begin{tabular}{|c|c|c|c|c|c|c|c|c|c|c|c|c|}
\hline & \multicolumn{6}{|c|}{ Rural women $(n 411)$} & \multicolumn{6}{|c|}{ Urban women ( $n$ 355) } \\
\hline & \multicolumn{3}{|c|}{2005} & \multicolumn{3}{|c|}{2010} & \multicolumn{3}{|c|}{2005} & \multicolumn{3}{|c|}{2010} \\
\hline & $\% *$ & Median & P25-P75 & $\%^{*}$ & Median & P25-P75 & $\%^{*}$ & Median & P25-P75 & $\%^{*}$ & Median & P25-P75 \\
\hline $\begin{array}{l}\text { Cooked porridge (e.g. maize meal, oats) and maize-based } \\
\text { drinks (\% of TE) }\end{array}$ & 100 & $40 \cdot 0$ & $29 \cdot 1-53.5$ & 100 & $32 \cdot 4$ & $19 \cdot 9-47 \cdot 3$ & 99 & $23 \cdot 4$ & $15 \cdot 6-32 \cdot 6$ & 100 & $18 \cdot 4$ & $9.8-28.9$ \\
\hline $\begin{array}{l}\text { Starchy grains (cereals, bread, vetkoek), starchy vegetables (potato, } \\
\text { sweet potato, corn) (\% of TE) }\end{array}$ & 100 & $24 \cdot 8$ & $16 \cdot 4-31.9$ & 100 & $19 \cdot 4$ & $13 \cdot 3-28 \cdot 4$ & 100 & $21 \cdot 6$ & $15 \cdot 9-28 \cdot 0$ & 100 & $21 \cdot 3$ & $15 \cdot 7-28 \cdot 3$ \\
\hline Fresh vegetables (\% of TE) & 100 & $1 \cdot 7$ & $1 \cdot 1-2 \cdot 5$ & 98 & 1.5 & $0.8-3 \cdot 0$ & 100 & 1.8 & $1 \cdot 3-2 \cdot 5$ & 100 & $2 \cdot 3$ & $1.4-3.4$ \\
\hline Fresh fruit and fruit juices (\% of TE) & 78 & 1.4 & $0.8-2.3$ & 90 & $2 \cdot 4$ & $1.0-4.9$ & 86 & $2 \cdot 8$ & $1 \cdot 6-4.7$ & 97 & 4.6 & $2 \cdot 6-8 \cdot 1$ \\
\hline Legumes and legume products (\% of TE) & 42 & $1 \cdot 2$ & $0.7-2 \cdot 1$ & 74 & 1.4 & $0.5-2.6$ & 66 & 0.9 & $0.5-1.7$ & 88 & 1.0 & $0.5-2 \cdot 1$ \\
\hline Nuts and seeds (\% of TE) & 18 & 2.5 & $1.3-4.4$ & 25 & 1.3 & $0.6-2.7$ & 48 & 1.4 & $0.8-2.5$ & 38 & 1.3 & $0.7-2.5$ \\
\hline Milk and milk products ( $\%$ of TE) & 52 & $2 \cdot 6$ & $1.4-4.8$ & 82 & $4 \cdot 3$ & $1 \cdot 8-7 \cdot 4$ & 95 & $4 \cdot 3$ & $2 \cdot 4-7 \cdot 0$ & 97 & 4.0 & $2 \cdot 4-6 \cdot 3$ \\
\hline All meat, chicken, fish, and egg and products ( $\%$ of TE) & 100 & $6 \cdot 6$ & $3 \cdot 9-10 \cdot 0$ & 99 & $7 \cdot 6$ & $0 \cdot 0-13 \cdot 1$ & 100 & $13 \cdot 8$ & $10 \cdot 1-17 \cdot 2$ & 100 & $13 \cdot 8$ & $9 \cdot 4-19$ \\
\hline All fats and oils and products (including ice cream) (\% of TE) & 99 & 4.7 & $2 \cdot 7-7 \cdot 3$ & 99 & $5 \cdot 2$ & $2 \cdot 7-10 \cdot 0$ & 100 & 7.9 & $5 \cdot 7-10.5$ & 100 & $5 \cdot 0$ & $3 \cdot 0-8 \cdot 6$ \\
\hline $\begin{array}{l}\text { Sugar, syrups, sweets and refined products with sugar added (sweet } \\
\text { cookies, cakes, biscuits) (\% of TE) }\end{array}$ & 99 & $6 \cdot 6$ & $3 \cdot 7-10 \cdot 4$ & 99 & $9 \cdot 2$ & $4 \cdot 7-16 \cdot 2$ & 100 & $9 \cdot 1$ & $6 \cdot 3-12 \cdot 7$ & 100 & 10.5 & $6 \cdot 3-17 \cdot 0$ \\
\hline Savoury snacks, dishes, sauces, seasoning and products ( $\%$ of TE) & 30 & 0.6 & $0 \cdot 1-2 \cdot 1$ & 75 & 0.6 & $0.2-1 \cdot 3$ & 88 & $2 \cdot 8$ & $0.9-5.5$ & 93 & $2 \cdot 4$ & $1 \cdot 1-4 \cdot 8$ \\
\hline Alcoholic drinks (\% of TE) & 25 & $9 \cdot 4$ & $3 \cdot 0-31 \cdot 3$ & 23 & $2 \cdot 6$ & $0.9-8.2$ & 44 & $9 \cdot 0$ & $4 \cdot 1-15 \cdot 9$ & 40 & 4.6 & $1 \cdot 2-9.9$ \\
\hline
\end{tabular}

PURE-NWP-SA, Prospective Urban and Rural Epidemiology in the North West Province of South Africa; P25, 25th percentile; P75, 75th percentile; TE, total energy.

*Percentage of consumers. 
Table 8 Percentage contribution to total energy intake of individual food groups eaten by at least $25 \%$ of male participants AND contributing at least $5 \%$ to total energy in 2005 or 2010 according to rural/urban residence; PURE-NWP-SA study

\begin{tabular}{|c|c|c|c|c|c|c|c|c|c|c|c|c|}
\hline & \multicolumn{6}{|c|}{ Rural men ( $n$ 186) } & \multicolumn{6}{|c|}{ Urban men ( $n$ 202) } \\
\hline & \multicolumn{3}{|c|}{2005} & \multicolumn{3}{|c|}{2010} & \multicolumn{3}{|c|}{2005} & \multicolumn{3}{|c|}{2010} \\
\hline & $\% *$ & Median & P25-P75 & $\% *$ & Median & P25-P75 & $\% *$ & Median & P25-P75 & $\% *$ & Median & P25-P75 \\
\hline Cooked porridge (e.g. maize meal, maltabella, oats) (\% of TE) & 99.5 & 35.09 & $24.51-47.23$ & $100 \cdot 0$ & 29.46 & $16 \cdot 43-45 \cdot 82$ & $100 \cdot 0$ & $26 \cdot 28$ & $18 \cdot 80-35 \cdot 39$ & 99.5 & $22 \cdot 88$ & $12.85-33.51$ \\
\hline Samp, maize rice, wheat rice, rice, macaroni, spaghetti (\% of TE) & $96 \cdot 2$ & 6.72 & $3 \cdot 88-9 \cdot 11$ & $96 \cdot 8$ & 3.39 & $1.74-5.55$ & 95.5 & 3.47 & $2 \cdot 22-5.55$ & $97 \cdot 0$ & 2.55 & $1.49-4.35$ \\
\hline Sugar, white and brown (\% of TE) & 95.7 & $5 \cdot 12$ & $2.93-7.85$ & $97 \cdot 3$ & 5.79 & $3.69-10.68$ & 98.5 & 4.47 & $2 \cdot 58-7.15$ & 97.5 & 4.24 & $2.15-7.91$ \\
\hline Bread, rolls, vetkoek (\% of TE) & 94.6 & $15 \cdot 32$ & $8.07-24 \cdot 83$ & 98.4 & 8.69 & $4 \cdot 79-17 \cdot 19$ & $97 \cdot 0$ & 14.61 & $8.58-21.47$ & $97 \cdot 0$ & 9.78 & $5.91-15 \cdot 90$ \\
\hline Beer, commercial and homemade (\% of TE) & 55.9 & 22.71 & $6.35-41.79$ & $51 \cdot 1$ & 9.79 & $3 \cdot 10-18.90$ & $69 \cdot 3$ & 11.67 & $5 \cdot 41-17 \cdot 63$ & 61.9 & 5.97 & $2.58-15.88$ \\
\hline
\end{tabular}

PURE-NWP-SA, Prospective Urban and Rural Epidemiology in the North West Province of South Africa; P25, 25th percentile; P75, 75th percentile; TE, total energy.

*Percentage of consumers.

Table 9 Percentage contribution to total energy intake of individual food groups eaten by at least $25 \%$ of female participants AND contributing at least $5 \%$ to total energy in 2005 or 2010 according to rural/urban residence; PURE-NWP-SA study

\begin{tabular}{|c|c|c|c|c|c|c|c|c|c|c|c|c|}
\hline & \multicolumn{6}{|c|}{ Rural women ( $n 411)$} & \multicolumn{6}{|c|}{ Urban women (n 355) } \\
\hline & \multicolumn{3}{|c|}{2005} & \multicolumn{3}{|c|}{2010} & \multicolumn{3}{|c|}{2005} & \multicolumn{3}{|c|}{2010} \\
\hline & $\%^{*}$ & Median & P25-P75 & $\% *$ & Median & P25-P75 & $\%^{*}$ & Median & P25-P75 & $\%^{*}$ & Median & P25-P75 \\
\hline Cooked porridge (e.g. maize meal, maltabella, & $100 \cdot 0$ & 38.73 & $8.22-53.17$ & $100 \cdot 0$ & 32.04 & $19 \cdot 17-47 \cdot 31$ & 99.4 & $23 \cdot 19$ & $15 \cdot 06-31 \cdot 74$ & $100 \cdot 0$ & 18.06 & $9.36-28.34$ \\
\hline Samp, maize rice, wheat rice, rice, macaroni, spaghetti (\% of TE) & $98 \cdot 8$ & $6 \cdot 78$ & $4.29-9.85$ & 98.1 & 3.96 & $2.04-5.95$ & $97 \cdot 2$ & 4.35 & $2.53-7.25$ & 98.9 & 3.35 & $2 \cdot 05-5 \cdot 2$ \\
\hline Sugar, white and brown ( $\%$ of TE) & $97 \cdot 3$ & 5.61 & $3.09-8.97$ & $96 \cdot 6$ & $6 \cdot 13$ & $2 \cdot 6-11.84$ & 99.8 & 4.94 & $3.07-7.53$ & 98.3 & $4 \cdot 37$ & $2 \cdot 06-8 \cdot 18$ \\
\hline Bread, rolls, vetkoek (\% of TE) & $95 \cdot 6$ & 14.68 & $8 \cdot 81-21.48$ & $96 \cdot 4$ & 8.57 & $4.31-14.71$ & $98 \cdot 0$ & $12 \cdot 83$ & $8.61-19.98$ & 97.7 & 9.43 & $5 \cdot 6-15 \cdot 07$ \\
\hline Beer, commercial and homemade (\% of TE) & $24 \cdot 3$ & $10 \cdot 18$ & $3.03-31.63$ & $23 \cdot 1$ & 2.57 & $0.89-8.61$ & 42.5 & $9 \cdot 16$ & $4 \cdot 32-16 \cdot 26$ & 37.5 & 4.61 & $1.34-9.87$ \\
\hline Maas/sour milk (\% of TE) & 1.5 & 6.91 & $1.08-10.98$ & 10.5 & $2 \cdot 68$ & $0.45-3.94$ & 33.5 & 0.99 & $0.51-1.91$ & $41 \cdot 1$ & 0.42 & $0.02-1 \cdot 16$ \\
\hline
\end{tabular}

PURE-NWP-SA, Prospective Urban and Rural Epidemiology in the North West Province of South Africa; P25, 25th percentile; P75, 75th percentile; TE, total energy.

*Percentage of consumers. 

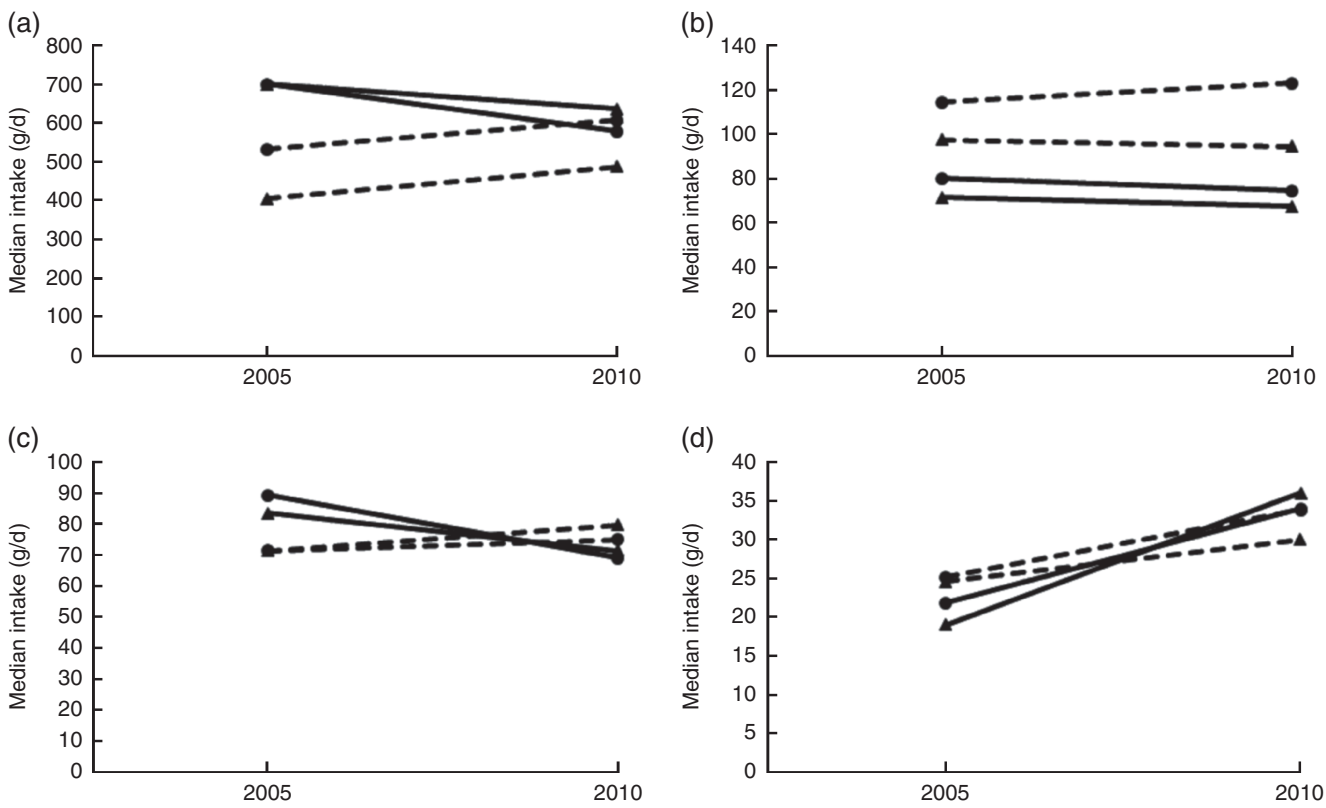

Fig. 2 Changes in the median intakes of four food groups over time (2005 to 2010) by men and women in the PURE-NWP-SA study: (a) cooked porridge; (b) bread and vetkoek; (c) samp, maize and rice; (d) white and brown sugar ( - urban men; $\_-$, rural women; - $-\_-$, urban women). PURE-NWP-SA, Prospective Urban and Rural Epidemiology in the North West Province of South Africa

Table 10 Baseline characteristics in 2005 of participants followed up from 2005 to 2010 (the cohort) compared with subjects lost to followup; PURE-NWP-SA study

\begin{tabular}{|c|c|c|c|c|c|c|c|}
\hline \multirow[b]{2}{*}{ Variable } & \multicolumn{3}{|c|}{$\begin{array}{l}\text { Baseline data in } 2005 \text { of subjects } \\
\text { followed up in } 2010\end{array}$} & \multicolumn{3}{|c|}{$\begin{array}{l}\text { Baseline data in } 2005 \text { of subjects lost to } \\
\text { follow-up in } 2010\end{array}$} & \multirow[b]{2}{*}{$P$ value* } \\
\hline & $n$ & Mean & SD & $n$ & Mean & SD & \\
\hline Age (years) & 1154 & $50 \cdot 8$ & $10 \cdot 3$ & 856 & 48.6 & $10 \cdot 4$ & $<0.001$ \\
\hline Sex (male; \%) & 1154 & \multicolumn{2}{|c|}{33.6} & 856 & \multicolumn{2}{|c|}{41.8} & $<0.001$ \\
\hline Locality (rural; \%) & 1154 & \multicolumn{2}{|c|}{$51 \cdot 7$} & 856 & \multicolumn{2}{|c|}{$47 \cdot 8$} & 0.080 \\
\hline Education attainment (no formal education; \%) & 1154 & \multicolumn{2}{|c|}{$36 \cdot 0$} & 856 & \multicolumn{2}{|c|}{$32 \cdot 1$} & 0.073 \\
\hline HIV positive (\%) & 1154 & \multicolumn{2}{|c|}{$17 \cdot 1$} & 856 & \multicolumn{2}{|c|}{$22 \cdot 2$} & 0.004 \\
\hline BMI $\left(\mathrm{kg} / \mathrm{m}^{2}\right)$ & 1154 & $25 \cdot 1$ & $7 \cdot 0$ & 853 & $24 \cdot 1$ & $7 \cdot 0$ & $<0.001$ \\
\hline Waist circumference $(\mathrm{cm})$ & 1147 & $80 \cdot 3$ & $12 \cdot 8$ & 843 & $79 \cdot 0$ & $13 \cdot 3$ & 0.007 \\
\hline HDL-cholesterol (mmol/l) & 1087 & 1.54 & 0.61 & 805 & 1.48 & 0.66 & 0.006 \\
\hline \multicolumn{8}{|l|}{ Energy intake $(\mathrm{MJ} / \mathrm{d})$} \\
\hline Median & 1154 & \multirow{2}{*}{\multicolumn{2}{|c|}{$\begin{array}{c}7 \cdot 4 \\
5 \cdot 6-10 \cdot 1\end{array}$}} & 796 & \multirow{2}{*}{\multicolumn{2}{|c|}{$\begin{array}{c}6 \cdot 9 \\
4 \cdot 7-9 \cdot 8\end{array}$}} & 0.003 \\
\hline P25-P75 & & & & & & & \\
\hline
\end{tabular}

PURE-NWP-SA, Prospective Urban and Rural Epidemiology in the North West Province of South Africa; P25, 25th percentile; P75, 75th percentile.

${ }^{*} P$ value for significant differences between subjects followed up in 2010 and those lost to follow-up ( $x^{2}$ test for categorical variables; Wilcoxon's signed-rank test for continuous variables).

increases in energy from protein and fat and decrease in energy from carbohydrate of all groups over time.

Tables 2 and 3 show that in this 5-year period from 2005 to 2010, rural and urban men and women decreased the contribution of cooked porridge and other starchy staples to total energy intake: for example, rural men and women had a median intake of cooked porridge of $700 \mathrm{~g} / \mathrm{d}$ in 2005 and decreased their intake to 621 and $650 \mathrm{~g} / \mathrm{d}$, respectively, in 2010. The corresponding figures for urban participants increased: urban women consumed $448 \mathrm{~g} / \mathrm{d}$ in 2005 and $507 \mathrm{~g} / \mathrm{d}$ in 2010, while urban men consumed $539 \mathrm{~g} / \mathrm{d}$ in 2005 and $679 \mathrm{~g} / \mathrm{d}$ in 2010. Given that energy intake of urban women increased from 9.0 to $11.7 \mathrm{MJ} / \mathrm{d}$ and that of urban men from 9.9 to $13.7 \mathrm{MJ} / \mathrm{d}$ (Tables 2 and $3)$, this explains the decreased energy contribution of cooked porridge to total energy intake from 36 to $30 \%$ in rural men, and from 26 to $23 \%$ in urban men, from 2005 to 2010 (Table 6). The corresponding figures were a decrease from 40 to $32 \%$ in rural women and from 23 to $18 \%$ in urban women over this 5 -year period (Table 7). However, cooked porridge remained the staple with almost all participants consuming it on a daily basis, as shown in Tables 2 to 5. Furthermore, Tables 4 and 5 also emphasize the decrease in consumption of the other starchy staple 
food group, which includes bread and vetkoek (a deepfried 'bun' made from fortified wheat flour), and especially the smaller contribution these foods made to total energy intake from 2005 to 2010, despite increased energy intakes over this time. The changes were from 22 to $20 \%$ in rural men; from 21 to $20 \%$ in urban men; from 25 to $19 \%$ in rural women; and from 22 to $21 \%$ in urban women (Tables 6 and 7). Therefore, although in both rural and urban participants the actual daily gram intake of starchy foods increased, the percentage contribution of staple foods to total energy intake decreased.

In addition, the change (2005 to 2010) in the gram intake (median) of cooked porridge was different between rural and urban areas for both men and women (men, $P=0.017$; women, $P<0.001)$. In 2005 , both men and women in rural areas consumed larger quantities of cooked porridge than their urban counterparts, while in 2010 it reversed (Fig. 2). For the samp, maize rice, etc. group, the change (2005 to 2010) in median intake was different between rural and urban areas (men, $P=0.012$; women, $P=0.005$ ). In 2005, both men and women in rural areas consumed more than their urban counterparts, while in 2010 it reversed. The median intake in 2005 and 2010 of bread and vetkoek was similar, but both men and women in urban areas consumed significantly more than their rural counterparts (men, $34 \mathrm{~g}$, $P=0.001$; women, $26 \mathrm{~g}, P<0.001)$.

Median vegetable, fruit, fruit juices and legume intakes increased in rural and urban men and women (Tables 2 and 3). However, total amounts consumed were relatively small. For example, median intake of fresh vegetables by rural women in 2010 was only $58 \mathrm{~g} / \mathrm{d}$ and in urban women $106 \mathrm{~g} / \mathrm{d}$.

Median consumption of milk and milk products by rural men increased from 8 to $110 \mathrm{~g} / \mathrm{d}$ and from 103 to $182 \mathrm{~g} / \mathrm{d}$ in urban men. The increases in rural women were from 8 to $111 \mathrm{~g} / \mathrm{d}$ and in urban women from 124 to $170 \mathrm{~g} / \mathrm{d}$ (Tables 2 and 3).

Foods containing added sugar increased from 25 to $102 \mathrm{~g} / \mathrm{d}$ in rural men from 2005 to 2010 , and from 85 to $141 \mathrm{~g} / \mathrm{d}$ in urban men over this period (Table 2). In women, the corresponding figures were from 33 to $101 \mathrm{~g} / \mathrm{d}$ and from 99 to $164 \mathrm{~g} / \mathrm{d}$ (Table 3). Tables 6 and 7 show that in all groups (except urban women) these figures represent less than $10 \%$ of total energy from added sugar in sugar-containing foods such as baked products, syrups and sweets. These figures could be misleading as this food group includes products like cake that is not only added sugar per se. However, in Tables 4 and 5, white and brown sugar consumption per se are shown (only the sugar added to tea, coffee, porridges, cold drinks, cake, etc.). This added sugar in the diet increased in all groups from 2005 to 2010, being consumed by more than $90 \%$ of all participants in 2005 and 2010. The median intake of sugar increased significantly by $12 \mathrm{~g} / \mathrm{d}$ for men in rural and urban areas between 2005 and $2010(P<0 \cdot 001)$. For women the change (2005 to 2010) in the median gram intake was different between rural and urban areas $(P=0.001)$. In 2005 , women in rural areas consumed less than their urban counterparts, while in 2010 it reversed (Fig. 2). The percentage of consumers in rural areas (men and women) of sugar-added carbonated cold drinks increased dramatically over time.

The intake of foods from animals (excluding milk and milk products) increased significantly from 2005 to 2010: from 48 to $97 \mathrm{~g} / \mathrm{d}$ in rural men, and from 131 to $191 \mathrm{~g} / \mathrm{d}$ in urban men (Table 2). In women, the corresponding figures were 45 to $78 \mathrm{~g} / \mathrm{d}$ for rural women and 120 to $174 \mathrm{~g} / \mathrm{d}$ for urban women (Table 3). However, Tables 4 and 5 show that of all the animal foods eaten, chicken was the most popular. More participants ate chicken than any other 'meat' in 2005 and this continued in 2010. Tables 4 and 5 also show that the changes in rural and urban men and women were essentially the same. Large increases were observed in the percentage of participants consuming processed meat over time in the rural men and women, while the percentage of consumers decreased for the urban groups. However, the quantities remained relatively small with the highest median intake of $21 \mathrm{~g} / \mathrm{d}$ (urban men in 2010).

From the above it becomes clear that the additional dietary energy in 2010 was provided by both 'healthier' and less 'healthier' food choices. Table 3, for example, shows that the 'healthier' food choices of women in 2010 were increased intakes of fresh vegetables, fresh fruit and juices, and legumes and legume products. The less 'healthier' choices were an increased consumption of more products containing sugar and savoury snacks and dishes. In men (Table 4), as in women (Table 5), marked increases in added sugar (sugar added to porridge or coffee and tea) were seen. A beneficial change seen in men (but not in women) was an increased intake of fresh, full-fat milk (Tables 4 and 5).

Another important observation from Tables 2 to 5 is that although the vegetable and fruit intakes in both men and women increased from 2005 to 2010, the total amounts of foods in these groups were relatively modest. For example, in 2010 rural women consumed a median of $\sim 58 \mathrm{~g}$ each of vegetables and fruit daily, while the urban women consumed 106 and $164 \mathrm{~g}$ of vegetables and fruit daily, respectively. This is still less than the recommendation of at least $400 \mathrm{~g} / \mathrm{d}$ given in the SAFBDG ${ }^{(27)}$.

The foods most often consumed included cooked porridge, sunflower oil, starchy grains, sugar, bread, coffee and tea, and some form of vegetables. The only animal protein food consumed by all groups as part of the 'top ten' consumed foods was chicken (either home-prepared or bought as fried chicken). Fruit was not part of the 'top ten' consumed foods for men, but white-flesh fruit (apples, bananas, pears) were for the urban women in 2005 and 2010 and the rural women in 2010.

Tables 6 and 7 show the percentage contribution to total energy intake of the twelve food groups. Cooked porridge 
stayed the main source of energy over time for both men and women in the rural and urban areas. The contribution of alcohol to energy intake decreased in all groups over time. Tables 8 and 9 show the percentage contribution to total energy intake of individual foods/food groups eaten by at least $25 \%$ of men and women and contributing at least $5 \%$ to total energy intake. In men (Table 8), only four individual food groups and alcoholic drinks complied with these criteria. In women (Table 9), the same individual food groups and alcoholic drinks met these criteria, as well as maas/sour milk (traditional fermented milk drink). It is noteworthy that the diet of the rural women in 2005 had the smallest variety of foods eaten in meaningful quantities. Table 11 presents the percentage of consumers who met the recommended number of portions according to the SAFBDG for vegetables and fruit as well as milk and milk products. Despite a significant increase from 2005 to 2010 in the number of participants meeting the recommendations and even after an improvement in energy intake, meaning that more foods were eaten in 2010 by rural and urban participants, less than a third of the men and women in urban areas met the recommendation for vegetables and fruit, and only $12.4 \%$ of urban men and $11.5 \%$ of urban women met the recommendation for milk and milk products.

\section{Discussion}

\section{Limitations and strengths of the study}

The main aim of this part of the PURE-NWP-SA study was to identify the foods and/or food groups responsible for the reported changes in nutrient intakes from 2005 to 2010 of the study participants ${ }^{(11)}$. A limitation of the study is the high proportion of subjects lost to follow-up, as discussed previously $^{(11,12)}$. Those lost to follow-up were significantly younger, more males, with a higher percentage of HIV infection, lower BMI, lower waist circumference, lower HDL-cholesterol and lower energy intake than those followed up. The main reasons were migration or death $^{(11,12)}$. Another limitation is that grouping of foods into only twelve food groups is tricky. Food products with different nutrient content, especially micronutrients, and weight per portion are grouped together and could be misleading. For example, the milk group contains liquid milk and cheese with well-known differences in quantities usually consumed. However, we continue to do it since we knew that the percentage of participants consuming cheese was relatively small and the portion size was also small. A potential limitation may be in the use of the QFFQ to measure individual dietary intake. However, the QFFQ was applied by well-trained fieldworkers in lengthy individual discussions of which foods were eaten during the previous month, how often, as well as an estimation of serving (portion) sizes. The QFFQ was extensively validated for this population for both individual nutrient and food intakes and as food groups ${ }^{(19,20,21,22)}$. The same

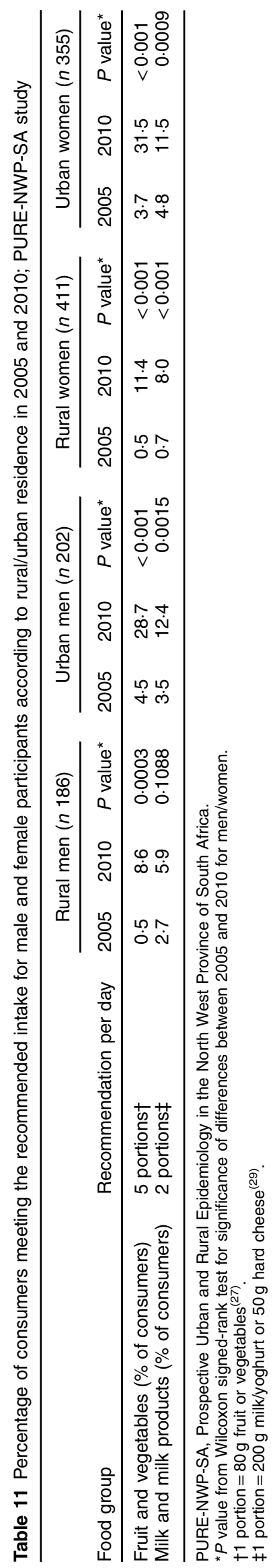


group of researchers and fieldworkers measured intakes in 2005 and 2010.

A major strength of the study is the prospective cohort design, although the period of only 5 years may be regarded as a limitation. However, our publication on nutrient intakes ${ }^{(11)}$ indicated significant differences in intakes during this 5-year period.

\section{The context of nutrient changes in evaluation of changes in food intake}

It has been mentioned that we found that the changes in energy and nutrient intakes of these rural and urban women and men from 2005 to 2010 followed the changes described for the nutrition transition in other parts of the world ${ }^{(1,11,12)}$. It is, however, important for developing policies and programmes for specific populations to steer the nutrition transition in a beneficial direction, to know which foods were responsible for positive changes, so that they may be targeted in interventions. Positive changes would be those that improve nutritional status (in this population particularly addressing micronutrient deficiencies), without increasing risk of overweight, obesity and therefore NCD. Briefly, taking the changes in intakes of women as an example, we showed ${ }^{(11)}$ that in 2005 rural women had a median energy intake of $6.2 \mathrm{MJ} / \mathrm{d}$ compared with $9 \cdot 0 \mathrm{MJ} / \mathrm{d}$ of urban women, a difference of $45 \%$. In 2010 this cohort of rural women increased their energy intake to $9 \cdot 1 \mathrm{MJ} / \mathrm{d}$ and the urban women to $11.7 \mathrm{MJ} / \mathrm{d}$, increases of 47 and $30 \%$, respectively. So, the rural women had an energy intake in 2010 similar to that of the urban women in 2005. Similarly, rural men increased their energy intake from a median of $6.9 \mathrm{MJ} / \mathrm{d}$ in 2005 to $9.7 \mathrm{MJ} / \mathrm{d}$ in 2010 and urban men from 9.9 to $13.7 \mathrm{MJ} / \mathrm{d}$ in the corresponding years (Table 2 and 3).

The changes in distribution of energy between the macronutrients gives a background for evaluating food contributions to changing energy intakes. Urban women had slightly more of their energy as total protein than rural women. Both urban and rural women ingested more of their energy as animal protein sources in 2005, and both rural and urban women decreased plant protein intake from a median of 7.5 and $6.1 \%$ of total energy in 2005 to 6.3 and $5.5 \%$, respectively, in 2010 . The changes in total fat intake are intriguing. In 2005, rural and urban women had respective median intakes of 20.3 and $28.4 \%$ of total energy as fat. In 2010, rural women had a median intake of $22.9 \%$ and urban women $27.7 \%$. It seems as if the urban women already reached a plateau in 2005 , while rural women had only a slight increase in total energy as fat. The differences and changes in saturated and polyunsaturated fats largely mirrored differences and changes of the animal $v$. plant protein intakes ${ }^{(11)}$.

It seems therefore that the percentage of energy as carbohydrate may reflect the biggest change in the distribution of energy. In 2005 , rural women had $66.7 \%$ of their energy as carbohydrate and urban women $55.7 \%$. In 2010 this changed to 61.7 and $54.1 \%$, respectively. Again, it seems that while the rural women decreased the total percentage of energy contributed by carbohydrate over the 5 years, the urban women may have reached a plateau or 'steady state' already in 2005 . However, the percentage of energy provided by added sugar increased in both the rural and urban women, from 6.4 to $8.9 \%$ and from 8.2 to $9.0 \%$, respectively, from 2005 to 2010 . Thus, although total carbohydrate energy decreased, energy from added sugar increased.

The changes observed in rural and urban men largely mirrored what was seen in women, although energy from total fat was even lower in the men ${ }^{(11)}$. Rural men had a median energy intake from fat in 2005 of $17.8 \%$ and of $21.5 \%$ in 2010 , while urban men increased their median energy intake from fat from 24.9 to $26 \cdot 3 \%$. These values are well below the value of $30 \%$ of energy from fat recommended in the SAFBDG ${ }^{(31)}$ and leaves room for increased energy intake from foods rich in $\alpha$-linoleic and $n$-3 fatty acids such as plant oils (e.g. sunflower or olive oil) or fish.

\section{Beneficial and detrimental changes in food intake} The results of the analysis of changes in foods eaten clearly showed some beneficial effects over the 5 years (more vegetables and fruit, more milk and milk products, more fish and more foods from animal sources), which will all contribute to improved micronutrient intakes. Unfortunately, these changes were small and did not allow the majority of participants to reach recommended intakes of vegetables, fruit, and milk and milk products. Furthermore, some detrimental changes were observed, especially an increase in added sugar intake, mainly in the form of sugar-sweetened beverages, increased consumption of beer by women and increased intakes of processed meat, savoury snacks and hard margarine (and therefore a higher saturated fat intake), illustrating an emergence of processed foods in the diet, which is of concern. We have previously shown that the increased intakes of added sugar and sucrose-sweetened beverages have a detrimental effect on several risk factors for $\mathrm{NCD}^{(12)}$ in this specific population. Furthermore, a study from Sweden showed that a sucrose intake of more than $15 \%$ of energy is associated with an increased risk of a coronary event ${ }^{(36)}$. Processed meat consumption has been linked to an increased risk of certain types of cancer $^{(37-40)}$. Hard margarines contribute to saturated fat intake and are potential sources of trans-fatty acids with known detrimental effects on risk of $\mathrm{CVD}^{(31)}$. More and more global dietary and food recommendations mention the need for home-prepared meals from fresh ingredients because of the high energy content of processed foods and links to obesity ${ }^{(41)}$. 


\section{Diet variety}

There was no evidence of 'new' foods eaten in 2010 and the 'top ten' consumed foods remained unchanged from 2005 to 2010. (Note: participants were always requested to report foods eaten that were not part of the QFFQ.) However, some products were consumed by a larger percentage of participants in 2010 than in 2005. For example, white-flesh fruit was eaten by $77.6 \%$ of rural women in 2005 and by $88.3 \%$ of them in 2010 . Similarly, more men consumed products such as milk, eggs, organ meats, processed meats, hard margarine and cooked starchy vegetables with added fat in 2010 than in 2005 .

\section{Dietary staples}

The decreased intake of cooked maize porridge and bread may be of concern because of the mandatory fortification of maize meal and bread flour ${ }^{(13)}$ to provide micronutrients. However, the data show that porridges are replaced to a certain extent by micronutrient-rich foods such as vegetables, fruit, milk and animal-origin foods. These trends should be encouraged to increase the total amounts of these foods eaten.

\section{Added sugar}

The increased intakes of products containing added sugar by all groups is a real concern that should be addressed. The South African Government is in a process of instituting taxation of sugar-sweetened beverages in an attempt to address the growing public health problem of obesity $^{(42,43)}$. Although this tax was introduced on 1 April 2018 , it is not certain if it will be an effective mechanism to address obesity in the South African population.

\section{Conclusions}

It is concluded that from 2005 to 2010, as part of the nutrition transition, rural and urban men and women in the NWP of SA changed dietary choices that resulted in improved diets, but also in some instances included more products that may increase risks of NCD. These changes were probably based on what were available and affordable to both rural and urban communities. The opening of a large food retail store in the rural community could have contributed to the availability and affordability of less healthy foods. This population needs to be informed on the potential effects of these potential negative dietary changes while encouraging current good practices like the consumption of whole grains, fruit and vegetables, chicken meat and canned fish. It is suggested that nutrition education interventions could assist the adult and adolescent populations in this province to choose their foods eaten based on knowledge of the beneficial and detrimental effects of certain foods and food products. Although this is the first prospective study in SA on a large cohort, the results should be interpreted with caution and not generalized for all the different ethnic groups.

\section{Acknowledgements}

Acknowledgements: The authors acknowledge the contribution of the late Professor Annamarie Kruger to the PURE South Africa study. They thank all supporting staff and participants in the PURE study; in particular, from PURE South Africa, the PURE-NWP-SA research team, fieldworkers and office staff in the Africa Unit for Transdisciplinary Health Research and the Centre of Excellence for Nutrition, Faculty of Health Sciences, North-West University, Potchefstroom, South Africa; and from PURE International, S. Yusuf and the PURE project office staff at the Population Health Research Institute, Hamilton Health Sciences and McMaster University, Ontario, Canada. Financial support: This work was supported by the South Africa Netherlands Research Program on Alternatives in Development; the South African National Research Foundation (GUN numbers 2069139 and FA200604070 0010); North-West University; the Population Health Research Institute, McMaster University, Canada; and the South African Medical Research Council. Funders were not involved in any aspect of the design, implementation, analysis, or interpretation and written account of the study. Conflict of interest: The authors declare that they have no competing interests. Authorship: E.W.-V. and H.H.V. were responsible for the conception and design, analysis and interpretation of the data and wrote the manuscript. R.L. was responsible for the statistical analysis of the data, made contributions to the interpretation of the data and critically revised the manuscript for important intellectual content. Ethics of buman subject participation: The Ethics Committee of the North-West University approved the study (04M10 and NWU-0016-10-A1). The study complied with all applicable requirements of international regulations, including the Helsinki Declaration of 1975 (as revised in 2008). Permission for the study was also obtained from the North-West Provincial Department of Health, local government authorities, and tribal chiefs in the rural areas. Volunteers gave written informed consent. The participants were transported to and from the study sites in both the urban and rural areas. They received lunch on site after blood sampling was completed. The study is registered at ClinicalTrials.gov (ID: NCT03225586; http://www.clinicaltrials.gov).

\section{References}

1. Hawkes C (2007) WHO Commission on Social Determinants of Health. Globalization, Food and Nutrition Transitions. Globalization and Health Knowledge Network: Research Papers. http://www.who.int/social_determinants/ resources/gkn_hawkes.pdf (accessed July 2018). 
2. Vorster HH, Kruger A \& Margetts BM (2011) The nutrition transition in Africa: can it be steered into a more positive direction? Nutrients 3, 429-441.

3. Mayosi BM, Flisher AJ, Lalloo UG et al. (2009) The burden of non-communicable diseases in South Africa. Lancet $\mathbf{3 7 4}$, 934-947.

4. Nojilana B, Bradshaw D, Pillay-van Wyk V et al. (2016) Persistent burden from non-communicable diseases in South Africa needs strong action. $S$ Afr Med $J$ 106, 436-437.

5. Shisana O, Labadarios D, Rehle T et al. (2013) South African National Health and Nutrition Examination Survey (SANHANES-1), pp. 135-144. Cape Town: HSRC Press.

6. Norman R, Bradshaw D, Schneider M et al. (2007) A comparative risk assessment for South Africa in 2000: towards promoting health and preventing disease. S Afr Med J 97, 637-641.

7. Vorster H, Venter C, Kruger H et al. (2000) The impact of urbanization on physical, physiological and mental health of Africans in the North West Province of South Africa: the THUSA study. S Afr J Sci 96, 505-514.

8. MacIntyre U, Kruger H, Venter C et al. (2002) Dietary intakes of an African population in different stages of transition in the North West Province, South Africa: the THUSA study. Nutr Res 22, 239-256.

9. Serfontein M, Venter C, Kruger A et al. (2010) Alcohol intake and micronutrient density in a population in transition: the Transition and Health during Urbanisation in South Africa (THUSA) study. S Afr J Clin Nutr 23, 22-28.

10. MacIntyre U, Venter C, Kruger A et al. (2012) Measuring micronutrient intakes at different levels of sugar consumption in a population in transition: the Transition and Health during Urbanisation in South Africa (THUSA) study. $S$ Afr J Clin Nutr 25, 122-130.

11. Wentzel-Viljoen E, Lee S, Laubscher R et al. (2018) Accelerated nutrition transition in the North West Province of South Africa: results from the Prospective Urban and Rural Epidemiology (PURE-NWP-SA) cohort study, 2005 to 2010. Public Health Nutr. Published online: 8 May 2018. doi: 10.1017/S1368980018001118.

12. Vorster HH, Kruger A, Wentzel-Viljoen E et al. (2014) Added sugar intake in South Africa: findings from the Adult Prospective Urban and Rural Epidemiology cohort study. Am J Clin Nutr 99, 1479-1486.

13. South African Department of Health (2003) Foodstuffs, Cosmetics and Disinfectants Act, 1972 (Act No. 54 of 1972). Regulations Relating to the Fortification of Certain Foodstuffs. Pretoria: Government Gazette, Republic of South Africa; available at https://extranet.who.int/nutrition/gina/ en/node/17871.

14. Van Heerden IV \& Schonfeldt HC (2011) The lack of food intake data and the consequences thereof: invited review. $S$ Afr J Clin Nutr 24, 10-18.

15. Vorster HH, Badham J \& Venter C (2013) An introduction to the revised food-based dietary guidelines for South Africa. $S$ Afr J Clin Nutr 26, Suppl, S5-S12.

16. Corsi DJ, Subramanian S, Chow CK et al. (2013) Prospective Urban Rural Epidemiology (PURE) study: baseline characteristics of the household sample and comparative analyses with national data in 17 countries. Am Heart $J \mathbf{1 6 6}$, 636-646.e4

17. Schutte AE, Schutte R, Huisman HW et al. (2012) Are behavioural risk factors to be blamed for the conversion from optimal blood pressure to hypertensive status in black South Africans? A 5-year prospective study. Int J Epidemiol 41, 1114-1123.

18. Sotunde O, Kruger H, Wright HH et al. (2015) Lean mass appears to be more strongly associated with bone health than fat mass in urban black South African women. J Nutr Health Aging 19, 628-636.
19. MacIntyre U, Venter C \& Vorster H (2001) A culturesensitive quantitative food frequency questionnaire used in an African population: 1 . Development and reproducibility. Public Health Nutr 4, 53-62.

20. MacIntyre U, Venter C, Vorster H et al. (2001) A combination of statistical methods for the analysis of the relative validation data of the quantitative food frequency questionnaire used in the THUSA study. Public Health Nutr $\mathbf{4}$, 45-51.

21. Wentzel-Viljoen E, Laubscher R \& Kruger A (2011) Using different approaches to assess the reproducibility of a culturally sensitive quantified food frequency questionnaire. $S$ Afr J Clin Nutr 24, 143-148.

22. MacIntyre U, Venter C \& Vorster H (2001) A culture-sensitive quantitative food frequency questionnaire used in an African population: 2. Relative validation by 7 -day weighed records and biomarkers. Public Health Nutr 4, 63-71.

23. Venter C, MacIntyre U \& Vorster H (2000) The development and testing of a food portion photograph book for use in an African population. I Hum Nutr Diet 13, 205-218.

24. Langenhoven M, Conradie P, Wolmarans P et al. (1991) MRC Food Quantities Manual. Cape Town: South African Medical Research Council.

25. Wolmarans P, Danster N, Dalton A et al. (2010) Condensed Food Composition Tables for South Africa. Cape Town: South African Medical Research Council.

26. Vorster HH (2013) 'Make starchy foods part of most meals': a food-based dietary guideline for South Africa. $S$ Afr J Clin Nutr 26, Suppl, S28-S35.

27. Naudé CE (2013) 'Eat plenty of vegetables and fruit every day': a food-based dietary guideline for South Africa. $S$ Afr J Clin Nutr 26, Suppl, S46-S56.

28. Venter CS, Ochse R \& Swart R (2013) 'Eat dry beans, split peas, lentils and soya regularly': a food-based dietary guideline. S Afr J Clin Nutr 26, Suppl, S36-S45.

29. Vorster HH, Wentzel-Viljoen E \& Vermaak M (2013) 'Have milk, maas or yoghurt every day': a food-based dietary guideline for South Africa. S Afr J Clin Nutr 26, Suppl., S57-S65.

30. Schonfeldt HC \& Hall N (2013) 'Fish, chicken, lean meat and eggs can be eaten daily': a food-based dietary guideline for South Africa. S Afr J Clin Nutr 26, Suppl., S66-S76.

31. Smuts CM \& Wolmarans P (2013) The importance of the quality or type of fat in the diet: a food-based dietary guideline for South Africa. S Afr J Clin Nutr 26, Suppl., S87-S99.

32. Temple NJ \& Steyn NP (2013) Sugar and health: a foodbased dietary guideline for South Africa. S Afr J Clin Nutr 26, Suppl, S100-S104.

33. Wentzel-Viljoen E, Steyn K, Ketterer E et al. (2013) 'Use salt and foods high in salt sparingly': a food-based dietary guideline for South Africa. S Afr J Clin Nutr 26, Suppl., S105-S113.

34. Jacobs L \& Steyn NP (2013) 'If you drink alcohol, drink sensibly.' Is this guideline still appropriate? S Afr J Clin Nutr 26, Suppl., 114-S119.

35. Faber M, Wenhold FA, MacIntyre UE et al. (2013) Presentation and interpretation of food intake data: factors affecting comparability across studies. Nutrition $\mathbf{2 9}$, 1286-1292.

36. Warfa K, Drake I, Wallström P et al. (2016) Association between sucrose intake and acute coronary event risk and effect modification by lifestyle factors: Malmö Diet and Cancer Cohort Study. Br J Nutr 116, 1611-1620.

37. Lippi G, Mattiuzzi C \& Cervellin G (2016) Meat consumption and cancer risk: a critical review of published metaanalyses. Crit Rev Oncol Hematol 97, 1-14.

38. Beaney AJ, Banim PJ, Luben R et al. (2017) Higher meat intake is positively associated with higher risk of developing 
pancreatic cancer in an age-dependent manner and are modified by plasma antioxidants: a prospective cohort study (EPIC-Norfolk) using data from food diaries. Pancreas $\mathbf{4 6}$, 672-678.

39. Crippa A, Larsson SC, Discacciati A et al. (2018) Red and processed meat consumption and risk of bladder cancer: a dose-response meta-analysis of epidemiological studies. Eur J Nutr 57, 689-701.

40. Stepien M, Chajes V \& Romieu I (2016) The role of diet in cancer: the epidemiologic link. Salud Publica Mex 58, 261273 .
41. Monteiro CA, Levy RB, Claro RM et al. (2010) Increasing consumption of ultra-processed foods and likely impact on human health: evidence from Brazil. Public Health Nutr 14, 5-13.

42. Tugendhaft A, Manyema M, Veerman LJ et al. (2016) Cost of inaction on sugar-sweetened beverage consumption: implications for obesity in South Africa. Public Health Nutr 19, 2296-2304.

43. Manyema M, Veerman LJ, Chola L et al. (2014) The potential impact of a $20 \%$ tax on sugar-sweetened beverages on obesity in South African adults: a mathematical model. PLoS One 9, e105287. 